\title{
Monopole Floer homology and Legendrian knots
}

\author{
STEVEn SiveK
}

\begin{abstract}
We use monopole Floer homology for sutured manifolds to construct invariants of unoriented Legendrian knots in a contact 3-manifold. These invariants assign to a knot $K \subset Y$ elements of the monopole knot homology $\operatorname{KHM}(-Y, K)$, and they strongly resemble the knot Floer homology invariants of Lisca, Ozsváth, Stipsicz, and Szabó. We prove several vanishing results, investigate their behavior under contact surgeries, and use this to construct many examples of nonloose knots in overtwisted 3-manifolds. We also show that these invariants are functorial with respect to Lagrangian concordance.
\end{abstract}

57M27, 57R58; 57R17

\section{Introduction}

A knot $\mathcal{K}$ in a contact 3 -manifold $(Y, \xi)$ is said to be Legendrian if the tangent vectors to $\mathcal{K}$ lie in the contact planes $\xi$. In recent years, a variety of invariants have been constructed to distinguish Legendrian knots which are topologically identical. Notable examples include contact homology as described by Eliashberg [11], especially the combinatorial version due to Chekanov [5], and invariant elements of knot Floer homology constructed by Ozsváth, Szabó and Thurston [36] using grid diagrams or by Lisca, Ozsváth, Stipsicz and Szabó [30] using open book decompositions; the last of these is often called the "LOSS invariant."

In order to construct a knot invariant from monopole Floer homology, Kronheimer and Mrowka [27] defined a monopole version of Juhász's sutured Floer homology [22] and declared the monopole knot homology $\operatorname{KHM}(Y, K)$ to be the sutured invariant of the complement of $K$. It is natural to ask whether an analogue of the LOSS invariant can be defined in this setting, where the construction makes no use of Heegaard diagrams or open books but instead proceeds by embedding the knot complement in a closed 3-manifold $\bar{Y}$ and computing $\overline{H M}(\bar{Y})$ in certain $\operatorname{Spin}^{c}$-structures.

The goal of this paper is to present such an invariant. Namely, to any unoriented Legendrian knot $\mathcal{K} \subset(Y, \xi)$ of topological type $K$, we associate elements

$$
\ell_{g}(\mathcal{K}) \in K H M(-Y, K)
$$


in monopole knot homology with local coefficients, which are invariant up to automorphisms of $K H M$, for all integers $g \geq 2$. (Conjecturally these do not depend on $g$, so for convenience we shall omit it throughout this introduction and the reader may fix any choice of $g$.) These elements are obtained by choosing a particular contact structure $\bar{\xi}$ on the closed manifold $\bar{Y}$, so that $\left(Y-\mathcal{K},\left.\xi\right|_{Y-\mathcal{K}}\right)$ is a contact submanifold of $(\bar{Y}, \bar{\xi})$, and letting $\ell_{g}(\mathcal{K})$ be the monopole contact invariant of $\bar{\xi}$.

The construction of $\ell(\mathcal{K})$ presents some advantages and disadvantages over that of the LOSS invariant. It is hard to compute in general, it does not distinguish between different orientations of $\mathcal{K}$, and it does not come with a natural bigrading the way elements of knot Floer homology do. However, some vanishing and nonvanishing results have very simple proofs, as do several theorems involving contact surgery. For example:

Proposition 4.1 If the complement of $\mathcal{K}$ is overtwisted, then $\ell(\mathcal{K})=0$.

Proposition 4.2 Let $S_{+}(\mathcal{K})$ and $S_{-}(\mathcal{K})$ denote the positive and negative stabilizations of a Legendrian knot $\mathcal{K}$. Then $\ell\left(S_{+} S_{-}(\mathcal{K})\right)=0$ for all $\mathcal{K}$.

For the LOSS invariant $\widehat{\mathcal{L}}(\mathcal{K})$ one can prove something stronger, namely, $\widehat{\mathcal{L}}\left(S_{-}(\mathcal{K})\right)=$ $\widehat{\mathcal{L}}(\mathcal{K})$ and $\hat{\mathcal{L}}\left(S_{+}(\mathcal{K})\right)=0$, but when working with unoriented knots one cannot distinguish between $S_{+}(\mathcal{K})$ and $S_{-}(K)$.

Theorem 5.1 Let $\mathcal{K}, \mathcal{S} \subset(Y, \xi)$ be disjoint Legendrian knots, and let $\mathcal{K}_{\mathcal{S}} \subset Y_{\mathcal{S}}$ denote the image of $\mathcal{K}$ in the contact manifold $Y_{\mathcal{S}}$ obtained by performing a contact $(+1)$-surgery on $\mathcal{S}$. Then there is a map

$$
\operatorname{KHM}(-Y, K) \rightarrow \operatorname{KHM}\left(-Y_{S}, K_{S}\right)
$$

sending $\ell(\mathcal{K})$ to $\ell\left(\mathcal{K}_{\mathcal{S}}\right)$.

These results are all known to be true for the LOSS invariant, as are several consequences we will pursue in this paper. However, using work of Mrowka and Rollin $[32 ; 33]$ on the monopole contact invariant which is not known to be true in Heegaard Floer homology, we can investigate one entirely new property of $\ell(\mathcal{K})$ : its behavior under Lagrangian concordance, as defined by Chantraine [4].

Theorem 6.3 Let $\mathcal{K}_{0}, \mathcal{K}_{1} \subset(Y, \xi)$ be Legendrian knots, with $Y$ a homology 3-sphere, and suppose that $\mathcal{K}_{0}$ is Lagrangian concordant to $\mathcal{K}_{1}$. Then there is a map

$$
\operatorname{KHM}\left(-Y, K_{1}\right) \rightarrow \operatorname{KHM}\left(-Y, K_{0}\right)
$$

such that $\ell\left(\mathcal{K}_{1}\right) \mapsto \ell\left(\mathcal{K}_{0}\right)$. 
The organization of this paper is as follows. In Section 2 we review the necessary background on sutured monopole homology and the monopole contact invariant. We construct $\ell_{g}(\mathcal{K})$, prove its invariance and compute it for Legendrian unknots in Section 3, and prove the vanishing theorems mentioned above in Section 4. In Section 5 we investigate the effect of contact $(+1)$-surgery on $\ell(\mathcal{K})$ and apply this to prove some nonvanishing results and to construct many examples of nonloose knots in overtwisted contact manifolds. Finally, in Section 6 we discuss the behavior of $\ell(\mathcal{K})$ with respect to Lagrangian concordance.

Throughout this paper we will adopt the convention that letters in the standard math font, such as $K$, refer to topological knots, whereas the same letters in a script font, such as $\mathcal{K}$, refer to Legendrian representatives of those knot types. We also remark that Lekili [29] has shown that one can replace $\widetilde{H M}$ with $\mathrm{HF}^{+}$in the Kronheimer-Mrowka construction of sutured monopole homology in order to recover sutured Floer homology. Thus the reader can apply the constructions in this paper to obtain a similar Legendrian invariant in knot Floer homology, and everything in this paper will still hold except the Lagrangian concordance results of Section 6. In this sense it would be interesting to compare $\ell(\mathcal{K})$ to the LOSS invariant.

Acknowledgement An early version of this work formed part of my thesis at MIT under the supervision of Tom Mrowka, who I thank for many useful discussions and suggestions. I am also grateful to many others, in particular John Baldwin, Jon Bloom, John Etnyre, Peter Kronheimer, Yank1 Lekili, Lenny Ng, Peter Ozsváth, Paul Seidel, Vera Vértesi and Chris Wendl, for helpful conversations on this work and related issues. I would also like to thank the anonymous referee for many helpful comments, and especially for raising the issue of orientation which was overlooked in the first version of this paper. This work was partially supported by an NSF Graduate Research Fellowship.

\section{Sutured manifolds and contact invariants in monopole Floer homology}

\subsection{The definition of $S H M$}

For background on monopole Floer homology we refer to Kronheimer and Mrowka's book [26].

Let $(M, \gamma)$ be a balanced sutured manifold. Kronheimer and Mrowka [27] defined the monopole Floer homology of $(M, \gamma)$ as follows: 
(1) Choose an oriented, connected surface $T$ such that the components of $\partial T$ are in one-to-one correspondence with the components of $\gamma$. Form the product sutured manifold $(T \times I, \delta)$, where $I=[-1,1]$, with annuli $A(\delta)=\partial T \times I$ and $R_{ \pm}(\delta)=T \times\{ \pm 1\}$.

(2) Glue the annuli $A(\delta)$ to $A(\gamma)$ by some orientation-reversing map $A(\delta) \rightarrow A(\gamma)$ sending $\partial R_{+}(\delta)$ to $\partial R_{+}(\gamma)$. The resulting 3-manifold should have boundary $\bar{R}_{+} \cup \bar{R}_{-}$for some connected, closed, orientable surfaces $\bar{R}_{ \pm}=R_{ \pm}(\gamma) \cup R_{ \pm}(\delta)$.

(3) Form a closed manifold $\bar{Y}$ by gluing the boundary along some diffeomorphism $h: \bar{R}_{+} \rightarrow \bar{R}_{-}$, and let $\bar{R} \subset \bar{Y}$ be the image of $\bar{R}_{ \pm}$.

We require that $\bar{R}$ has genus at least 2, and that $T$ contains a simple closed curve $c$ such that $c \times\{ \pm 1\}$ is a nonseparating curve in $\bar{R}_{ \pm}$.

Definition 2.1 The sutured monopole homology of $(M, \gamma)$ is defined as

$$
\operatorname{SHM}(M, \gamma)=\widetilde{H M}_{\bullet}(\bar{Y} \mid \bar{R}),
$$

where $\widetilde{H M} \bullet(\bar{Y} \mid \bar{R})$ is the direct sum of $\widetilde{H M} \bullet(\bar{Y}, \mathfrak{s})$ over all $\operatorname{Spin}^{c}$ structures $\mathfrak{s}$ satisfying $\left\langle c_{1}(\mathfrak{s}), \bar{R}\right\rangle=2 g(\bar{R})-2$.

Note that since $g(\bar{R}) \geq 2$, the class $c_{1}(\mathfrak{s})$ cannot be torsion if $\overline{H M}_{\bullet}(\bar{Y}, \mathfrak{s})$ contributes to $\widetilde{H M}_{\bullet}(\bar{Y} \mid \bar{R})$; but then $\overline{H M}(Y, \mathfrak{s})=0$, so $\widetilde{H M}_{\bullet}(\bar{Y}, \mathfrak{s})$ and $\widehat{H M} \bullet(\bar{Y}, \mathfrak{s})$ are canonically isomorphic. In [27] the authors therefore simply write $H M(\bar{Y} \mid \bar{R})$, but we will prefer to leave the "to" decoration in place as a reminder that we will be using the contact invariant associated to $\widetilde{H M}$.

We can also define $S H M$ using local coefficients. Let $\mathcal{R}$ be a ring with exponential map exp: $\mathbb{R} \rightarrow \mathcal{R}^{\times}$and write $t^{n}=\exp (n)$ for convenience. To any smooth $1-$ cycle $\eta$ in $\bar{Y}$ we can associate a local system $\Gamma_{\eta}$ on the Seiberg-Witten configuration space $\mathcal{B}(\bar{Y}, \mathfrak{s})$ whose fiber at any point is $\mathcal{R}$ and which assigns to any path $z:[0,1] \rightarrow \mathcal{B}(\bar{Y}, \mathfrak{s})$ the multiplication map by $t^{r(z)}$, where

$$
r(z)=\frac{i}{2 \pi} \int_{[0,1] \times \eta} \operatorname{tr}\left(F_{A_{z}}\right)
$$

for $A_{z}$ the connection on $[0,1] \times \bar{Y}$ arising from the path $z$.

Suppose that the diffeomorphism $h: \bar{R}_{+} \rightarrow \bar{R}_{-}$restricts to an orientation-preserving homeomorphism $c \times\{1\} \rightarrow c \times\{-1\}$, resulting in a curve $\bar{c} \subset \bar{Y}$. If $\eta$ is taken to be a curve dual to $\bar{c}$, in the sense that $\bar{c} \cdot \eta=1$, then we can define $\operatorname{SHM}\left(M, \gamma ; \Gamma_{\eta}\right)=$ $\widetilde{H M}_{\bullet}\left(\bar{Y} \mid \bar{R} ; \Gamma_{\eta}\right)$. As in the case without local coefficients, if $t-t^{-1}$ is invertible in $\mathcal{R}$ then the authors simply write $\operatorname{HM}\left(\bar{Y} \mid \bar{R} ; \Gamma_{\eta}\right)$ without any ambiguity but we will continue to use $\widetilde{H M}$. 
Proposition 2.2 [27] If $t-t^{-1}$ is invertible in $\mathcal{R}$, then $\operatorname{SHM}\left(M, \gamma ; \Gamma_{\eta}\right)$ depends only on $(M, \gamma)$ and $\mathcal{R}$. In this case we can allow $\bar{R}$ to have genus 1 , but if $g(\bar{R}) \geq 2$ and $\mathcal{R}$ has no $\mathbb{Z}$-torsion then we also have

$$
\operatorname{SHM}\left(M, \gamma ; \Gamma_{\eta}\right) \cong \operatorname{SHM}(M, \gamma) \otimes \mathcal{R} .
$$

\subsection{SHM with coefficients in $\mathbb{Z} / 2 \mathbb{Z}$}

Throughout [27] the authors work with coefficients (both local and otherwise) in $\mathbb{Z}$; however, we assert that $S H M$ is still an invariant if $\mathbb{F}=\mathbb{Z} / 2 \mathbb{Z}$ is used instead. When using systems of local coefficients $\Gamma_{\eta}$ over $\mathbb{F}$, we drop the condition that the ring $\mathcal{R}$ have no $\mathbb{Z}$-torsion and thus only require that $t-t^{-1} \in \mathcal{R}$ be invertible. This will allow us to pursue several applications involving surgery exact triangles, which are known to work with local coefficients over $\mathbb{F}$ (see [28] or [26, Chapter 42]) but which have not yet been proved with local coefficients over $\mathbb{Z}$.

The proofs of the invariance theorems in [27], Theorem 4.4 and Proposition 4.6, rely on several facts, most notably the excision theorems, Theorems 3.1 through 3.3, which still apply verbatim. We need to verify that a handful of important proofs still work, and in each case the only step requiring some additional care is the vanishing of a Tor group coming from an application of the Künneth theorem:

Corollary [27, Corollary 3.4] Let $\Sigma \subset Y$ be a closed, oriented surface, and let $\eta$ be a 1-cycle supported in $\Sigma$. If every component of $\Sigma$ has genus at least 2, then

$$
\widetilde{H M}\left(Y \mid \Sigma ; \Gamma_{\eta}\right) \cong \widetilde{H M}(Y \mid \Sigma) \otimes \mathcal{R} .
$$

Proof The only detail requiring care in the original proof is the map (14), denoted

$$
H_{\bullet}\left(Y_{1} \mid \Sigma_{1} ; \Gamma_{\eta_{0}}\right) \otimes H M_{\bullet}\left(Y_{2} \mid \Sigma_{2}\right) \rightarrow H M_{\bullet}\left(\tilde{Y} \mid \tilde{\Sigma} ; \Gamma_{\eta_{0}}\right),
$$

which comes from an application of the Künneth theorem and is expected to be an isomorphism. The cokernel of this map is

$$
\operatorname{Tor}_{\mathbb{F}}\left(H M_{\bullet}\left(Y_{1} \mid \Sigma_{1} ; \Gamma_{\eta_{0}}\right), H M_{\bullet}\left(Y_{2} \mid \Sigma_{2}\right)\right),
$$

which is zero since $H_{\bullet}\left(Y_{1} \mid \Sigma_{1} ; \Gamma_{\eta_{0}}\right)=\mathcal{R}$ is a free $\mathbb{F}$-module, so the rest of the proof still applies.

Lemma [27, Lemma 4.7] Let $Y$ be fibered over $S^{1}$ with closed fiber $R$ of genus at least 2. Then $\widetilde{H M}(Y \mid R) \cong \mathbb{F}$. 
Proof As in the above proof of [27, Corollary 3.4], if $Y_{h}$ is the mapping torus of $h: R \rightarrow R$ then $\widetilde{H M}\left(Y_{h} \mid R\right) \cong \widetilde{H M}\left(Y_{h^{-1}} \mid R\right)$ and so the excision theorem [27, Theorem 3.1] applied to $Y_{h} \sqcup Y_{h^{-1}}$ gives an injective map $\widetilde{H M}\left(Y_{h} \mid R\right) \otimes_{\mathbb{F}} \widetilde{H M}\left(Y_{h} \mid R\right) \rightarrow \mathbb{F}$ with cokernel

$$
\operatorname{Tor}_{\mathbb{F}}\left(\widetilde{H M}\left(Y_{h} \mid R\right), \widetilde{H M}\left(Y_{h} \mid R\right)\right) \text {. }
$$

Since $\widetilde{H M}\left(Y_{h} \mid R\right)$ is a free $\mathbb{F}$-module, this Tor term vanishes and the map is an isomorphism, hence $\widetilde{H M}\left(Y_{h} \mid R\right) \cong \mathbb{F}$.

Corollary [27, Corollary 4.8] The sutured homology group $\operatorname{SHM}(M, \gamma)$ does not depend on the choice of gluing homeomorphism $h$.

Proof This is another application of the excision theorem [27, Theorem 3.1] to a disconnected manifold $Y=Y_{1} \sqcup Y_{2}$ with $Y_{2}$ a mapping torus, hence $\widetilde{H M}\left(Y_{2} \mid \Sigma_{2}\right) \cong \mathbb{F}$ and again the proof is the same once we observe that

$$
\operatorname{Tor}_{\mathbb{F}}\left(\widetilde{H M}\left(Y_{1} \mid \Sigma_{1}\right), \widetilde{H M}\left(Y_{2} \mid \Sigma_{2}\right)\right) \cong 0
$$

Proposition [27, Proposition 4.10] If $t-t^{-1}$ is invertible in $\mathcal{R}$, then $\operatorname{SHM}\left(M, \gamma ; \Gamma_{\eta}\right)$ is independent of the genus $g$.

Proof Here we wish to show that

$$
\widetilde{H M}\left(Y_{1} \mid \bar{R}_{1} ; \Gamma_{\eta_{1}}\right) \cong \widetilde{H M}\left(\left(Y_{1} \sqcup Y_{2}\right) \mid\left(\bar{R}_{1} \sqcup \bar{R}_{2}\right) ; \Gamma_{\eta}\right)
$$

where $\eta=\eta_{1}+\eta_{2}$ for some cycles $\eta_{i} \subset \bar{R}_{i} \subset Y_{i}$, and we know $\widetilde{H M}\left(Y_{2} \mid \bar{R}_{2} ; \Gamma_{\eta_{2}}\right)=\mathcal{R}$. The Künneth theorem thus gives a map

$$
\widetilde{H M}\left(Y_{1} \mid \bar{R}_{1} ; \Gamma_{\eta_{1}}\right) \otimes_{\mathcal{R}} \mathcal{R} \rightarrow \widetilde{H M}\left(\left(Y_{1} \sqcup Y_{2}\right) \mid\left(\bar{R}_{1} \sqcup \bar{R}_{2}\right) ; \Gamma_{\eta}\right)
$$

with cokernel

$$
\operatorname{Tor}_{\mathcal{R}}\left(\widetilde{H M}\left(Y_{1} \mid \bar{R}_{1} ; \Gamma_{\eta_{1}}\right), \mathcal{R}\right)
$$

and since $\mathcal{R}$ is free as an $\mathcal{R}$-module, the Tor term vanishes and this is indeed an isomorphism.

We conclude that both the standard and local versions of sutured monopole homology are invariants if we work over $\mathbb{F}$ rather than $\mathbb{Z}$. 


\subsection{Monopole knot homology}

Given a knot $K$ in a closed, oriented 3-manifold $Y$, we can form a sutured manifold $Y(K)=(M, \gamma)$ following Juhász [22] by taking $M$ to be the knot complement $Y \backslash N(K)$ and $\gamma \subset \partial M$ a pair of oppositely oriented meridians. Then monopole knot homology is defined by

$$
\operatorname{KHM}(Y, K)=\operatorname{SHM}(M, \gamma),
$$

and if we work with local coefficients we get $\operatorname{KHM}(Y, K) \otimes \mathcal{R} \cong \operatorname{SHM}\left(M, \gamma ; \Gamma_{\eta}\right)$. From now on we will fix $\mathcal{R}$ to be the Novikov ring

$$
\left\{\sum_{\alpha} c_{\alpha} t^{\alpha} \mid \alpha \in \mathbb{R}, c_{\alpha} \in \mathbb{F}, \#\left\{\beta<n \mid c_{\beta} \neq 0\right\}<\infty \text { for all } n\right\},
$$

with $\exp (\alpha)=t^{\alpha}$ and $\left(t-t^{-1}\right)^{-1}=-t-t^{3}-t^{5}-\cdots$. Although we may drop the local system $\Gamma_{\eta}$ from our notation, we are always working with local coefficients over $\mathcal{R}$.

\subsection{Contact structures in monopole Floer homology}

Let $(Y, \xi)$ be a closed contact 3-manifold. Kronheimer and Mrowka [25] associate a contact invariant

$$
\psi(\xi): \Lambda(\xi) \rightarrow \widetilde{H M}_{\bullet}\left(-Y, \mathfrak{s}_{\xi}, c_{\text {bal }}, \Gamma_{\eta}\right)
$$

where $c_{\text {bal }}=2 \pi c_{1}\left(\mathfrak{s}_{\xi}\right)$ is a balanced perturbation of the Seiberg-Witten equations and $\Lambda(\xi)$ is the set of orientations of an appropriate moduli space. In general we will ignore the orientations $\Lambda(\xi)$, since we are working in characteristic 2 , and so we will write $\psi(\xi) \in \widetilde{H M}_{\bullet}\left(-Y, \mathfrak{s}_{\xi}, c_{\text {bal }}, \Gamma_{\eta}\right)$.

Mrowka and Rollin $[32 ; 33]$ investigated the behavior of the contact invariant under symplectic cobordisms.

Definition 2.3 A symplectic cobordism $(W, \omega)$ from $\left(Y_{-}, \xi_{-}\right)$to $\left(Y_{+}, \xi_{+}\right)$is said to be left-exact if $\omega$ is exact near $Y_{-}$, or equivalently if it is given in a collar neighborhood of $Y_{-}$by a symplectization $\frac{1}{2} d\left(t^{2} \eta_{-}\right)$where $\xi_{-}=\operatorname{ker} \eta_{-}$. It is right-exact if the same holds near $Y_{+}$, and boundary-exact if it is both left- and right-exact.

Theorem 2.4 [33, Theorem 3.5.4] Let $W$ be a boundary-exact cobordism $(W, \omega)$ as above such that the map

$$
H^{1}(W ; \mathbb{Z}) \rightarrow H^{1}\left(Y_{+} ; \mathbb{Z}\right)
$$

is surjective, and let $W^{\dagger}$ denote $W$ viewed as a cobordism from $-Y_{+}$to $-Y_{-}$. Then $\psi\left(\xi_{-}\right)=\widetilde{H M}\left(W^{\dagger}, \mathfrak{s}_{\omega}\right)\left(\psi\left(\xi_{+}\right)\right)$. 
Corollary 2.5 If $W$ is a symplectic 2-handle cobordism corresponding to Legendrian surgery, then

$$
\psi\left(\xi_{-}\right)=\widetilde{H M}\left(W^{\dagger}\right)\left(\psi\left(\xi_{+}\right)\right)
$$

and in particular we can replace the map $\widetilde{H M}\left(W^{\dagger}, \mathfrak{s}_{\omega}\right)$ of Theorem 2.4 by the total map $\overline{H M}\left(W^{\dagger}\right)$.

Remark 2.6 The results in this section are stated for monopole Floer homology in [33], which is not publicly available as of the time of this writing. However, the necessary excision theorems for the Seiberg-Witten moduli spaces which define the cobordism maps are proved in [32], where they are interpreted in terms of the equality of certain integer-valued Seiberg-Witten invariants rather than the equality of elements of the Floer homology group $\widetilde{H M}$. The main difficulty in extending these theorems to $\widetilde{H M}$ lies in orienting these moduli spaces compatibly, but since we work in characteristic 2 this will not be an issue.

\section{The Legendrian knot invariant}

Let $\mathcal{K} \subset(Y, \xi)$ be a Legendrian knot of topological knot type $K$. Our goal is to construct an appropriate contact structure $\bar{\xi}$ on a closure $(\bar{Y}, \bar{R})$ of the sutured knot complement $Y(K)$ so that the contact invariant $\psi(\bar{\xi})$ does not depend on any of the choices we must make. This will give us an invariant $\ell(\mathcal{K})$ of the Legendrian knot $\mathcal{K}$ which is an element of $\operatorname{KHM}(-Y, K)$ up to automorphism.

Take a standard neighborhood $N(\mathcal{K})$ whose boundary is a convex torus. If we assign coordinates to $\partial N(\mathcal{K}) \cong \mathbb{R}^{2} / \mathbb{Z}^{2}$ so that $( \pm 1,0)$ is a meridian and $(0, \pm 1)$ a longitude, then its dividing set $\Gamma$ consists of two parallel curves of slope $1 / \operatorname{tb}(\mathcal{K})$, where $\operatorname{tb}(\mathcal{K})$ is the Thurston-Bennequin invariant of $\mathcal{K}$. (See for example Etnyre and Honda [15, Section 2.4].) In particular, if we view the sutured knot complement $Y(\mathcal{K})$ as the contact manifold $\left(Y \backslash N(\mathcal{K}),\left.\xi\right|_{Y \backslash N(\mathcal{K})}\right)$ with convex boundary, then each of the meridional sutures will intersect each dividing curve transversely in a single point as in Figure 1. Here, and in all other figures, we will color regions white and gray to represent the positive and negative regions, respectively, of a convex surface.

\subsection{Closure of the sutured knot complement}

Our construction follows the definition of the sutured invariant as in Section 2. We must first pick an auxiliary surface $T$ whose boundary components are in one-toone correspondence with the sutures of $Y(\mathcal{K})$ and glue the annuli $\partial T \times I \subset T \times I$ to neighborhoods $A(\gamma)$ of the sutures. In order to form a contact structure on this 


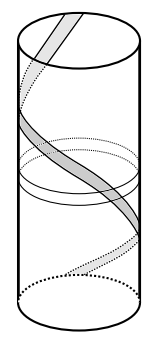

Figure 1. The convex torus $\partial(Y \backslash N(\mathcal{K}))$, cut along a meridian. The horizontal circles are sutures, while the pair of parallel arcs (or circles, once the top and bottom are identified) are dividing curves and have slope $1 / \mathrm{tb}(\mathcal{K})$.

glued manifold, we must assign a contact structure to $T \times I$ whose restriction to a neighborhood of $\partial T \times I$ agrees with $\xi$ in a neighborhood of $A(\gamma)$. By Giroux's flexibility theorem [19] it suffices to ensure that the dividing curves match, sending the positive region of $A(\gamma)$ to the negative region of $\partial T \times I$ and vice versa.

Let $T_{0}$ be a closed surface of genus at least 2, and pick a pair of dual curves $\alpha, \beta \subset T_{0}$ such that $\alpha \cdot \beta=1$. Give $T_{0} \times I$ the $I$-invariant contact structure $\Xi_{\alpha}$ whose dividing curves consist of two parallel disjoint copies of $\alpha$ on each surface $T_{0} \times\{ \pm 1\}$ and for which the negative region of $T_{0} \times\{+1\}$ is an annulus. Such a contact structure always exists: see eg Honda, Kazez and Matić [21, Theorem 1.1, case 3]. We define $(T \times I, \Xi)$ as the contact manifold obtained by cutting $T_{0} \times I$ along a convex perturbation of the annulus $\beta \times I$, as in Figure 2 .

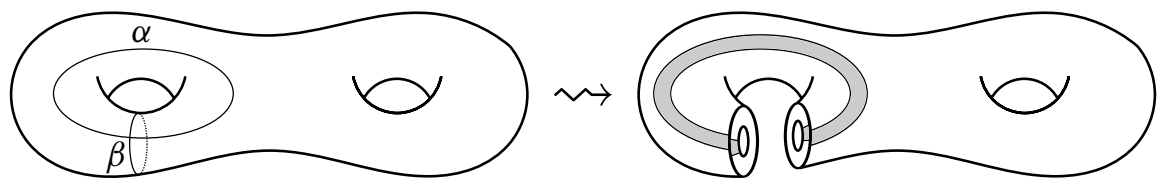

Figure 2. The construction of the auxiliary surface $(T \times I, \Xi)$

We now form a contact manifold $\left(Y^{\prime}, \xi^{\prime}\right)=(Y \backslash N(\mathcal{K})) \cup(T \times I)$ by gluing along some orientation-reversing diffeomorphism $A(\delta) \rightarrow A(\gamma)$ as described above. This manifold has edges, corresponding to the corners $\partial T \times \partial I$, which we smooth using edge-rounding as in Honda [20], under which dividing curves turn to the left (as viewed from outside $Y^{\prime}$ ) as they approach an edge. See Figure 3.

Lemma 3.1 The contact manifold $Y^{\prime}=(Y \backslash N(\mathcal{K})) \cup(T \times I)$ depends only on $\mathcal{K}$, $(Y, \xi)$ and the genus of $T_{0}$. 

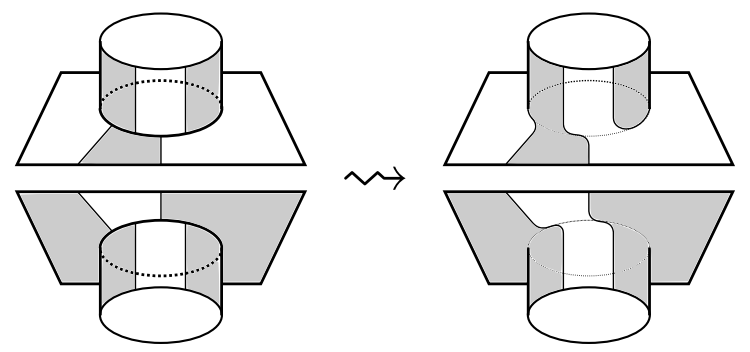

Figure 3. Gluing $T \times I$ to $Y \backslash N(\mathcal{K})$ and rounding edges in a cylindrical neighborhood of one of the sutures on $\partial N(\mathcal{K})$, as viewed from $T \times\{1\}$ on top and $T \times\{-1\}$ on the bottom

Proof The construction of $T \times I$ depends only on $g\left(T_{0}\right)$ and on the curves $\alpha, \beta \subset T_{0}$. Given any other pair of curves $\alpha^{\prime}$ and $\beta^{\prime}$ which intersect once, there is a diffeomorphism $\varphi: T_{0} \rightarrow T_{0}$ with $\varphi(\alpha)=\alpha^{\prime}$ and $\varphi(\beta)=\varphi\left(\beta^{\prime}\right)$, and this extends to a contactomorphism $\varphi \times$ Id: $\left(T_{0} \backslash N(\beta)\right) \times I \rightarrow\left(T_{0} \backslash N\left(\beta^{\prime}\right)\right) \times I$.

Finally, we close up $Y^{\prime}$ to get a contact manifold $(\bar{Y}, \bar{\xi})$ with distinguished convex surface $\bar{R}$ of genus $g \geq 2$. The boundary of $Y^{\prime}$ consists of two convex surfaces $\bar{R}_{+}$ and $\bar{R}_{-}$determined by $T \times\{ \pm 1\} \subset \bar{R}_{ \pm}$. These are split by pairs of parallel dividing curves $\Gamma_{ \pm} \subset \bar{R}_{ \pm}$into positive and negative regions $\left(\bar{R}_{+}\right)_{ \pm} \subset \bar{R}_{+}$and $\left(\bar{R}_{-}\right)_{ \pm} \subset \bar{R}_{-}$, and each of $\left(\bar{R}_{+}\right)_{-}$and $\left(\bar{R}_{-}\right)_{+}$is an annulus. Fix any diffeomorphism $h: \bar{R}_{+} \rightarrow \bar{R}_{-}$ which sends $\left(\bar{R}_{+}\right)_{ \pm}$to $\left(\bar{R}_{-}\right)_{\mp}$, and hence also $\Gamma_{+}$to $\Gamma_{-}$, and such that $h(x \times\{1\})$ and $x \times\{-1\}$ lie in the same component of $\Gamma_{-}$for any $x \times\{1\}$ in $\Gamma_{+} \cap(\operatorname{int}(T) \times\{1\})$. In other words, a dividing curve $c \subset \Gamma_{+}$corresponds to one of the two copies of $\alpha \subset T_{0}$, and we require $h(c)$ to be the dividing curve of $\Gamma_{-}$corresponding to the same copy of $\alpha$.

We glue $\bar{R}_{+}$to $\bar{R}_{-}$via $h$. The resulting contact manifold is the desired $(\bar{Y}, \bar{\xi})$.

Definition 3.2 The Legendrian invariant of $\mathcal{K}$ is defined as $\ell(\mathcal{K})=\psi(\bar{Y}, \bar{\xi}) \in$ $\widetilde{H M}\left(-\bar{Y}, \mathfrak{s} \bar{\xi} ; \Gamma_{\eta}\right)$.

We can compute that

$$
\left\langle c_{1}(\bar{\xi}), \bar{R}\right\rangle_{\bar{Y}}=\chi\left(\left(\bar{R}_{+}\right)_{+}\right)-\chi\left(\left(\bar{R}_{+}\right)_{-}\right)=2-2 g(\bar{R})
$$

and so $\left\langle c_{1}\left(\mathfrak{s}_{\bar{\xi}}\right), \bar{R}\right\rangle_{-\bar{Y}}=2 g(\bar{R})-2$. This means that $\ell(\mathcal{K})$ is in fact an element of $\widetilde{H M}\left(-\bar{Y} \mid \bar{R} ; \Gamma_{\eta}\right)=\operatorname{SHM}\left(-Y(K) ; \Gamma_{\eta}\right)$, which is by definition the knot homology with local coefficients. Therefore

$$
\ell(\mathcal{K}) \in K H M(-Y, K) \otimes \mathcal{R} .
$$


Remark 3.3 The desire to arrange that $\left\langle c_{1}(\bar{\xi}), \bar{R}\right\rangle=2-2 g$ motivated our choice of contact structure on $T \times I$. In particular, $\chi\left(\left(\bar{R}_{+}\right)_{+}\right)$and $\chi\left(\left(\bar{R}_{+}\right)_{-}\right)$sum to $\chi\left(\bar{R}_{+}\right)=$ $2-2 g$, and if we fix their difference as above then we must have $\chi\left(\left(\bar{R}_{+}\right)_{-}\right)=0$. But now $\left(\bar{R}_{+}\right)_{-}$does not have any sphere or torus components, and if it had disk components then $\bar{R}_{+}$would not have a tight neighborhood [20], so $\left(\bar{R}_{+}\right)_{-}$is forced to be a union of annuli.

In addition to the Legendrian knot $\mathcal{K} \subset(Y, \xi)$, the construction of $\ell(\mathcal{K})$ from a closure $(\bar{Y}, \bar{\xi})$ with distinguished convex surface $\bar{R}$ potentially depends on both the genus $g=g(\bar{R})$ and the choice of diffeomorphism $\bar{R}_{+} \rightarrow \bar{R}_{-}$. Our goal in the next section is to prove that in fact it is independent of the diffeomorphism.

\subsection{Invariance under diffeomorphism}

In this section we establish that $\ell(\mathcal{K})$ is independent of the choice of diffeomorphism $\bar{R}_{+} \rightarrow \bar{R}_{-}$.

Proposition 3.4 Let $\left(\bar{Y}^{\prime}, \bar{\xi}^{\prime}\right)$ be the contact manifold obtained from $\bar{Y}$ by cutting along the convex surface $\bar{R}$ and regluing along some orientation-preserving diffeomorphism $h$ such that $h(\gamma)=\gamma$ for each dividing curve $\gamma$ of $\bar{R}$. Then there is an isomorphism $\widetilde{H M}\left(-\bar{Y}^{\prime} \mid \bar{R} ; \Gamma_{\eta}\right) \rightarrow \widetilde{H M}\left(-\bar{Y} \mid \bar{R} ; \Gamma_{\eta}\right)$ which sends $\psi\left(\bar{Y}^{\prime}, \bar{\xi}^{\prime}\right)$ to $\psi(\bar{Y}, \bar{\xi})$.

Lemma 3.5 Proposition 3.4 holds when $h$ is a Dehn twist along some nonseparating curve $c$ which does not intersect the dividing curves $\Gamma$ of $\bar{R}$.

Proof We observe that $c$ is nonisolating, ie that every component of $\bar{R} \backslash(\Gamma \cup c)$ has a boundary component which intersects $\Gamma$, and thus by the Legendrian Realization Principle (see Kanda [24] and Honda [20]) we can take $c$ to be Legendrian. Indeed, the complement $\bar{R} \backslash \Gamma$ has two connected components; if $c$ is nonseparating within its component then it is clearly nonisolating. Otherwise, $c$ divides its component of $\bar{R} \backslash \Gamma$ into two components, say $A$ and $B$. Since $c$ is nonseparating in $\bar{R}$ there is a path in $\bar{R} \backslash c$ which connects $A$ to $B$, and this path must pass through the other component of $\bar{R} \backslash \Gamma$. In particular, the path crosses $\Gamma$, so both $\partial A$ and $\partial B$ intersect $\Gamma$ and thus $c$ is nonisolating.

Suppose now that $h$ is a positive Dehn twist along $c$, and that $c$ has been realized as a Legendrian curve. Then $h$ can be realized by $(-1)$-surgery on $c$ with respect to the framing induced by $\bar{R}$, and since $\operatorname{tw}(c, \bar{R})=-\frac{1}{2}|c \cap \Gamma|=0$ this is a Legendrian surgery. If $W$ is the corresponding symplectic cobordism, and $W^{\dagger}$ is the oppositely oriented cobordism from $-\bar{Y}^{\prime}$ to $-\bar{Y}$, then

$$
\widetilde{H M}\left(W^{\dagger}\right)\left(\psi\left(\bar{Y}^{\prime}, \bar{\xi}^{\prime}\right)\right)=\psi(\bar{Y}, \bar{\xi})
$$


by Corollary 2.5. The fact that $\widetilde{H M}\left(W^{\dagger}\right)$ gives an isomorphism $\widetilde{H M}\left(-\bar{Y}^{\prime} \mid \bar{R} ; \Gamma_{\eta}\right) \rightarrow$ $\widehat{H M}\left(-\bar{Y} \mid \bar{R} ; \Gamma_{\eta}\right)$ is an easy consequence of the surgery exact triangle for $\overline{H M}$ and the fact that $\bar{R}$ becomes compressible in the manifold $-\bar{Y}_{0}$ obtained by 0 -surgery along $c$, hence $\widetilde{H M}\left(-\bar{Y}_{0} \mid \bar{R}\right)=0$ by the adjunction inequality [26].

If instead $h$ is a negative Dehn twist, we note that $\bar{Y}$ can be obtained from $\bar{Y}$, by a positive Dehn twist along $c$, so we construct a cobordism $W$ from $\bar{Y}^{\prime}$ to $\bar{Y}$ as above and then $\widetilde{H M}\left(W^{\dagger}\right)^{-1}$ is the desired isomorphism.

Proof of Proposition 3.4 In general, we can arrange by an isotopy that the diffeomorphism $h$ is actually the identity on each dividing curve. Then $h$ restricts to a boundary-fixing diffeomorphism on the closure of each component of $\bar{R} \backslash \Gamma$. One component is an annulus $A$, so up to isotopy $\left.h\right|_{A}$ is a composition of Dehn twists about the core of $A$. The other component is a surface $\Sigma$ of genus $g(\bar{R})-1 \geq 1$ with two boundary components, and so $\left.h\right|_{\Sigma}$ can also be expressed as a product of Dehn twists about nonseparating curves which do not intersect $\Gamma=\partial \Sigma$. Since $h=\left.\left.h\right|_{A} \circ h\right|_{\Sigma}$, repeated application of Lemma 3.5 completes the proof of Proposition 3.4.

We have now shown that the construction of $\ell(\mathcal{K}) \in \operatorname{KHM}(-Y, K) \otimes \mathcal{R}$ is independent of all choices except possibly the genus $g=g(\bar{R})$. Thus we have constructed a sequence of Legendrian knot invariants $\ell_{g}(\mathcal{K})$ for $g \geq 2$.

Conjecture 3.6 The elements $\ell_{g}(\mathcal{K}), g \geq 2$, are all equal up to automorphism as elements of $\operatorname{KHM}(-Y, K) \otimes \mathcal{R}$.

Since we will show in Section 3.3 that $\ell_{g}(\mathcal{U})=1 \in \mathcal{R}$ where $\mathcal{U} \subset\left(S^{3}, \xi_{\text {std }}\right)$ is the Legendrian unknot with $t b=-1$, this conjecture would follow from a connected sum formula of the form

$$
\ell_{g}(\mathcal{K}) \otimes \ell_{g^{\prime}}(\mathcal{U})=\ell_{g+g^{\prime}-1}(\mathcal{K} \# \mathcal{U})=\ell_{g+g^{\prime}-1}(\mathcal{K})
$$

which we expect to be true by comparison with the LOSS invariant [30, Theorem 7.1]. From now on we will drop the $g$ subscript where convenient and simply write $\ell(\mathcal{K})$ to mean $\ell_{g}(\mathcal{K})$ for some fixed $g \geq 2$.

\subsection{The Legendrian unknot}

The Legendrian representatives of the topological unknot $U \subset S^{3}$ were classified by Eliashberg and Fraser [12]: they are completely determined by their classical invariants tb and $r$, and there is a representative $\mathcal{U}$ with $(\mathrm{tb}, r)=(-1,0)$ so that all others are 
stabilizations of $\mathcal{U}$. In this subsection we will prove that the Legendrian invariant of $\mathcal{U}$ is a unit of $K H M(U) \otimes \mathcal{R} \cong \mathcal{R}$.

Our strategy is to explicitly determine the contact structure on a particular closure $\bar{Y}$ of $S^{3}(\mathcal{U})$.

Lemma 3.7 Let $\xi$ be the $I$-invariant contact structure on $\left(S^{1} \times I\right) \times I$ whose dividing curves on the annulus $S^{1} \times I$ are a pair of parallel $\operatorname{arcs}\left\{t_{1}\right\} \times I$ and $\left\{t_{2}\right\} \times I$. Then after edge-rounding, $\xi$ is contactomorphic to the complement of $\mathcal{U}$.

Proof By Honda's classification of tight contact structures on solid tori [20, Theorem 2.3], there is a unique tight contact structure $\Xi$ on $S^{1} \times D^{2}$ for which the dividing curves on the boundary have slope -1 ; since $\operatorname{tb}(\mathcal{U})=-1$, the complement of $\mathcal{U}$ must be $\left(S^{1} \times D^{2}, \Xi\right)$. But if we round the edges on $\left(\left(S^{1} \times I\right) \times I, \xi\right)$, we get a tight contact structure on $S^{1} \times D^{2}$ for which the dividing curves on the boundary $S^{1} \times S^{1}$ have slope -1 , and so this contact structure must be $\Xi$ as well.

Proposition 3.8 The invariant $\ell(\mathcal{U})$ is a unit in $K H M(U) \otimes \mathcal{R} \cong \mathcal{R}$.

Proof We glue a surface $T \times I$ to $\left(S^{1} \times I\right) \times I$ as in Section 3.1, identifying the annuli $\partial T \times I$ with $\left(S^{1} \times I\right) \times \partial I$, to get an $I$-invariant contact manifold $Y^{\prime}=\Sigma_{g} \times I$ with convex boundary which is tight by Giroux's criterion for tightness [20, Theorem 3.5], which says that an $\mathbb{R}$-invariant contact structure on $\Sigma_{g} \times \mathbb{R}(g>0)$ is tight if and only if the dividing set on $\Sigma_{g} \times\{0\}$ has no contractible components. Gluing $\Sigma_{g} \times\{1\}$ to $\Sigma_{g} \times\{-1\}$ via the identity map, we get the closure $\bar{Y}=\Sigma_{g} \times S^{1}$ with $S^{1}$-invariant, tight contact structure $\bar{\xi}$ and distinguished surface $\bar{R}=\Sigma_{g} \times\{*\}$. Since no component of $\Gamma \subset \Sigma_{g}$ is separating, [34, Theorem 5] asserts that $\bar{\xi}$ is weakly fillable.

The claim that $\operatorname{KHM}(U) \otimes \mathcal{R}=\widetilde{H M}\left(\bar{Y} \mid \bar{R} ; \Gamma_{\eta}\right) \cong \mathcal{R}$ now follows from [27, Corollary 2.3]. Furthermore, since $\bar{\xi}$ is weakly fillable we know that $\psi(\bar{\xi})$ is a unit of $\widetilde{H M}\left(\bar{Y} ; \Gamma_{\eta}\right)[25 ; 33]$, and since $\psi(\bar{\xi}) \in \widetilde{H M}\left(\bar{Y} \mid \bar{R} ; \Gamma_{\eta}\right) \cong \mathcal{R}$ the proposition follows.

Remark 3.9 Wendl [40, Corollary 2] has shown that $(\bar{Y}, \bar{\xi})$ has vanishing untwisted ECH contact invariant. By work of Taubes [39] it follows that the untwisted contact invariant $\psi(\bar{\xi}) \in \widetilde{H M}(\bar{Y} \mid \bar{R})$ is also zero, so we must work with local coefficients for $\ell(\mathcal{U})$ to be nonzero.

We can also compute $\ell\left(\mathcal{U}_{Y}\right)$ if $\mathcal{U}_{Y}$ is a Legendrian unknot in a Darboux ball of some contact manifold $(Y, \xi)$. Observe that both $S^{3}(U)$ and $S^{3}(1)$ have $\left(\Sigma_{g} \times S^{1}, \Sigma_{g} \times\{*\}\right)$ as a closure, where $M(1)$ denotes the complement of a ball in $M$ with a single suture, and since $Y\left(U_{Y}\right) \cong Y \# S^{3}(U)$ we conclude that $\operatorname{KHM}\left(Y, U_{Y}\right) \cong \operatorname{SHM}(Y(1))$. 
Let $\widetilde{H M}(Y)=\operatorname{SHM}(Y(1)) \cong \operatorname{KHM}\left(Y, U_{Y}\right)$. Then clearly $\widetilde{H M}$ is analogous to the hat version of Heegaard Floer homology, since $\widehat{H F}(Y) \cong S F H(Y(1))$ virtually by definition [22]. In fact, it is equivalent to define $\overparen{H M}(Y)$ as the homology of the mapping cone of $U_{\dagger}: \check{C}(Y) \rightarrow \check{C}(Y)$ (see Bloom, Mrowka, and Ozsváth [1]), just as $\widehat{H F}(Y)$ comes from the Heegaard Floer complex $C F^{+}(Y)$.

We will define a contact invariant $\widetilde{\psi}_{g}(\xi) \in \widetilde{H M}(-Y) \otimes \mathcal{R}$ up to automorphism as $\ell_{g}\left(\mathcal{U}_{Y}\right)$. (Having noted that $\widetilde{\psi}_{g}$ potentially depends on $g$ just as $\ell_{g}$ does, we will similarly drop the subscript and write $\widetilde{\psi}(\xi)$ from now on.) This seems to be a reasonable choice by analogy with the LOSS invariant $\widehat{\mathcal{L}}\left(\mathcal{U}_{Y}\right) \in \widehat{H F K}\left(-Y, U_{Y}\right)$, which is identified with the Heegaard Floer contact invariant $\widehat{c}(\xi) \in \widehat{H F}(-Y)$ as argued in the proof of [30, Corollary 7.3].

Proposition 3.10 There is a map

$$
\widetilde{H M}(-Y) \otimes_{\mathbb{F}} \mathcal{R} \rightarrow \widetilde{H M}(-Y) \otimes_{\mathbb{F}} \mathcal{R}
$$

which sends $\tilde{\psi}(\xi)=\ell\left(\mathcal{U}_{Y}\right)$ to $\psi(\xi) \otimes 1$.

Proof Recall that $\mathcal{U} \subset\left(S^{3}, \xi_{\text {std }}\right)$ has closure $(\bar{Y}, \bar{R})=\left(\Sigma_{g} \times S^{1}, \Sigma_{g} \times\{*\}\right)$ with $S^{1}$-invariant contact structure $\bar{\xi}$ and its homology is twisted by a 1 -cycle $\eta \subset \bar{Y}$. Thus the Legendrian unknot $\mathcal{U}_{Y} \subset Y$ has closure $(Y \# \bar{Y}, \bar{R})$.

Build a symplectic cobordism $(W, \omega)$ from $(Y, \xi) \sqcup(\bar{Y}, \bar{\xi})$ to $(Y \# \bar{Y}, \xi \# \bar{\xi})$ by attaching a symplectic $1-$ handle to the symplectization $(Y \sqcup \bar{Y}) \times I$. The induced map

$$
\widetilde{H M}\left(W_{\dagger}, \mathfrak{s}_{\omega}\right): \widetilde{H M}\left(-(Y \# \bar{Y}), \mathfrak{s}_{\xi} \# \mathfrak{s}_{\bar{\xi}} ; \Gamma_{\eta}\right) \rightarrow \widetilde{H M}\left(-Y \sqcup-\bar{Y}, \mathfrak{s}_{\xi} \sqcup \mathfrak{s}_{\bar{\xi}} ; \Gamma_{\eta}\right)
$$

sends $\ell\left(\mathcal{U}_{Y}\right)=\psi(\xi \# \bar{\xi}) \in \widetilde{H M}\left(-(Y \# \bar{Y}) ; \Gamma_{\eta}\right)$ to $\psi(\xi \sqcup \bar{\xi})$ by Theorem 2.4. But the map

$$
\widetilde{H M}\left(-Y, \mathfrak{s}_{\xi}\right) \otimes_{\mathbb{F}} \widetilde{H M}\left(-\bar{Y}, \mathfrak{s}_{\bar{\xi}} ; \Gamma_{\eta}\right) \rightarrow \widetilde{H M}\left(-Y \sqcup-\bar{Y}, \mathfrak{s}_{\xi} \sqcup \mathfrak{s}_{\bar{\xi}} ; \Gamma_{\eta}\right)
$$

coming from the Künneth theorem is an isomorphism since $\widetilde{H M}\left(-\bar{Y}, \mathfrak{s}_{\bar{\xi}} ; \Gamma_{\eta}\right) \cong \mathcal{R}$ is free, so in fact $\widetilde{H M}\left(W_{\dagger}, \mathfrak{s}_{\omega}\right)$ can be expressed as a map

$$
\widetilde{H M}\left(-(Y \# \bar{Y}), \mathfrak{s}_{\xi} \# \mathfrak{s}_{\bar{\xi}} ; \Gamma_{\eta}\right) \rightarrow \widetilde{H M}\left(-Y, \mathfrak{s}_{\xi}\right) \otimes_{\mathbb{F}} \mathcal{R}
$$

sending $\psi(\xi \# \bar{\xi})$ to $\psi(\xi) \otimes \psi(\bar{\xi})=\psi(\xi) \otimes 1$. The source and target of this map are summands of $\widetilde{H M}\left(-(Y \# \bar{Y}) \mid \bar{R} ; \Gamma_{\eta}\right)=\widetilde{H M}(-Y) \otimes \mathcal{R}$ and $\widetilde{H M}(-Y) \otimes \mathcal{R}$, respectively, and $\psi(\xi \# \bar{\xi})$ is $\ell\left(\mathcal{U}_{Y}\right)$, so we are done.

Corollary 3.11 If $\psi(\xi) \in \widetilde{H M}(-Y)$ is nonzero, then so is $\widetilde{\psi}(\xi) \in \widetilde{H M}(-Y) \otimes \mathcal{R}$.

For example, if $\xi$ is strongly symplectically fillable then $\psi(\xi)$ is nonzero and primitive [25; 32], so Proposition 3.10 implies that $\widetilde{\psi}(\xi)$ is a primitive element of $\widetilde{H M}(-Y) \otimes \mathcal{R}$. 


\section{Vanishing results}

\subsection{Loose knots}

Recall that a Legendrian knot $\mathcal{K} \subset(Y, \xi)$ is said to be loose if the complement of $\mathcal{K}$ is overtwisted.

Proposition 4.1 If $\mathcal{K} \subset Y$ is loose, then $\ell(\mathcal{K})=0$.

Proof By assumption $Y \backslash \mathcal{K}$ has an overtwisted disk, so any closure $(\bar{Y}, \bar{\xi})$ does as well. Then $\psi(\bar{Y}, \bar{\xi})$ vanishes (see [32, Corollary B]), hence $\ell(\mathcal{K})$ does as well.

\subsection{Stabilization}

Let $S_{+}(\mathcal{K})$ and $S_{-}(\mathcal{K})$ denote the positive and negative stabilizations of an oriented Legendrian knot $\mathcal{K}$, which may also be thought of as the connected sums $\mathcal{K} \# \mathcal{U}_{ \pm}$where $\mathcal{U}_{ \pm} \subset S^{3}$ is the topologically trivial knot with $\mathrm{tb}=-2$ and $r= \pm 1$. One would like to show that $\ell\left(S_{-}(\mathcal{K})\right)=\ell(\mathcal{K})$ and $\ell\left(S_{+}(\mathcal{K})\right)=0$ for any Legendrian knot $\mathcal{K}$, since the same holds for the LOSS invariant $\hat{\mathcal{L}}(\mathcal{K})$ [30]. Unfortunately, this is impossible, since one can only distinguish between these stabilizations when $\mathcal{K}$ is oriented.

On the other hand, the double stabilization $S_{+} S_{-}(\mathcal{K})$ is still well-defined and unambiguous even for unoriented $\mathcal{K}$, since the Legendrian isotopy type of $\mathcal{U}_{+} \# \mathcal{U}_{-}$is preserved by orientation reversal, and one can prove the corresponding result in this case.

Proposition 4.2 If $\mathcal{K}$ is any Legendrian knot, then $\ell\left(S_{+} S_{-}(\mathcal{K})\right)=0$.

Proof We will construct a closure $\bar{Y}$ of $\mathcal{K}^{\prime}=S_{+} S_{-}(\mathcal{K})$ with an overtwisted disk, so that the vanishing follows from [32, Corollary B]. Stabilization of a Legendrian knot $\mathcal{K}$ corresponds to attaching a bypass to its complement: if we stabilize to get $S_{ \pm}(\mathcal{K})$ inside a standard neighborhood $N(\mathcal{K}) \subset Y$ and fix a standard neighborhood $N\left(\mathcal{K}^{ \pm}\right) \subset N(\mathcal{K})$, then $Y \backslash N\left(\mathcal{K}^{ \pm}\right)$is obtained from $Y \backslash N(\mathcal{K})$ by a bypass attachment. See Etnyre and Honda [14] or the proof of [38, Theorem 1.5] by Stipsicz and Vértesi for discussion.

In the leftmost part of Figure 4 we have indicated the attaching $\operatorname{arcs} c_{+}$and $c_{-}$of bypasses corresponding to positive and negative stabilizations on $\partial\left(Y \backslash N\left(\mathcal{K}^{\prime}\right)\right)$, as in [38, Figure 10], with a single meridional suture $\mu$ between them. The dividing curves are shown with orientation for convenience, so that they have the same orientation as the boundary $\partial \Gamma_{+}$of the positive region. If we start to form the closure of $Y \backslash N\left(\mathcal{K}^{\prime}\right)$ by 


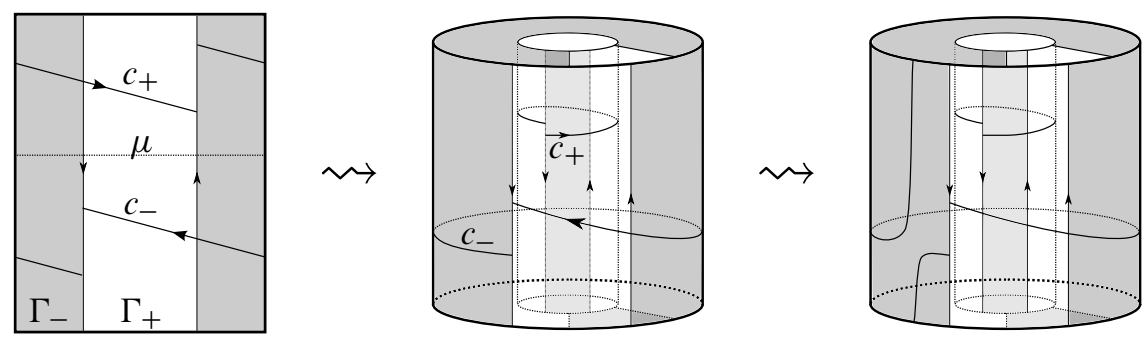

Figure 4. Attaching curves for bypasses in the complement of $S_{+} S_{-}(\mathcal{K})$ and its closure

attaching a surface $T \times I$ to neighborhoods of the sutures and then rounding edges, we may then cut out $T \times I$ to get a contact manifold with corners as in the middle figure; this indicates the positions of the arcs $c_{ \pm}$on the boundary components $\bar{R}_{ \pm} \subset Y^{\prime}$.

We wish to glue $\bar{R}_{+}$to $\bar{R}_{-}$so that the arcs $c_{+}$and $c_{-}$are glued together, but as shown in the middle of Figure 4 we cannot do this by identifying the inside and outside regions in the obvious way. Indeed, we must identify the white component $\left(\bar{R}_{+}\right)_{+}$on the outside with the gray component $\left(\bar{R}_{-}\right)_{-}$on the inside, identifying the left dividing curve on the outside with the left dividing curve on the inside and likewise for the right dividing curves, but then $c_{+}$and $c_{-}$cannot be made parallel so that they end up identified. The problem is that as we follow them leftward and around the back of the cylinder from the leftmost dividing curves, the arc $c_{-}$ends "above" its starting point whereas $c_{+}$ends "below" its starting point. However, we can glue $c_{+}$to $c_{-}$by applying a Dehn twist to the outer gray annulus $\left(\bar{R}_{+}\right)_{-}$along its core as shown on the right side of Figure 4 . We can then "untwist" $c_{-}$by reparametrizing $\left(\bar{R}_{+}\right)_{-}$, sliding the lower endpoint of $c_{-}$downward along its dividing curve until it has nearly traversed the entire curve and lies just above the other endpoint; this allows us to identify it with $\left(\bar{R}_{-}\right)_{+}$so that $c_{-}$is sent to $c_{+}$. Now we can glue $\bar{R}_{+}$to $\bar{R}_{-}$so that $c_{-}$and $c_{+}$ are identified, and the union of their respective bypasses is an overtwisted disk in the closure $(\bar{Y}, \bar{\xi})$. We conclude that $\psi(\bar{Y}, \bar{\xi})$, and hence $\ell\left(\mathcal{K}^{\prime}\right)$, is zero.

\section{Contact surgery}

\subsection{Behavior under contact $(+1)-$ surgery}

The following is a direct analogue of Theorem 1.1 of Ozsvatth and Stipsicz [35], which concerns the LOSS invariant $\widehat{\mathcal{L}}(\mathcal{K}) \in \widehat{H F K}(-Y, K)$ (or more generally $\mathcal{L}(\mathcal{K}) \in$ $\left.\operatorname{HFK}^{-}(-Y, K)\right)$ but is much harder to prove. 
Theorem 5.1 Let $\mathcal{K}$ and $\mathcal{S}$ be disjoint Legendrian knots in $(Y, \xi)$, and let $\left(Y_{\mathcal{S}}, \xi_{\mathcal{S}}\right)$ denote the contact manifold obtained by performing contact $(+1)$-surgery along $\mathcal{S}$. Let $\mathcal{K}_{\mathcal{S}}$ be the image of the Legendrian knot $\mathcal{K}$ in $Y_{\mathcal{S}}$. Then there is a map

$$
\operatorname{KHM}(-Y, K) \otimes \mathcal{R} \rightarrow \operatorname{KHM}\left(-Y_{\mathcal{S}}, K_{\mathcal{S}}\right) \otimes \mathcal{R}
$$

such that $\ell(\mathcal{K}) \mapsto \ell\left(\mathcal{K}_{\mathcal{S}}\right)$.

Proof We may obtain $(Y, \xi)$ by performing contact $(-1)$-surgery on $\mathcal{S} \subset Y_{\mathcal{S}}$ (see Ding and Geiges [8, Proposition 8]). Since $\mathcal{S}$ and $\mathcal{K}$ are disjoint it is easy to see that we can fix a closure $\bar{Y}_{\mathcal{S}}$ of the complement $Y_{\mathcal{S}}\left(\mathcal{K}_{\mathcal{S}}\right)$ so that contact $(-1)$-surgery on $\mathcal{S} \subset \bar{Y}_{\mathcal{S}}$ gives a closure $\bar{Y}$ of $Y(\mathcal{K})$, and the surface $\bar{R}$ and cycle $\eta \subset \bar{R}$ are the same in both closures. The Weinstein cobordism $(W, \omega)$ from $\bar{Y}_{\mathcal{S}}$ to $\bar{Y}$ coming from this handle attachment gives a map

$$
\widetilde{H M}\left(W^{\dagger}\right): \widetilde{H M}\left(-\bar{Y} ; \Gamma_{\eta}\right) \rightarrow \widetilde{H M}\left(-\bar{Y}_{\mathcal{S}} ; \Gamma_{\eta}\right)
$$

carrying $\ell(\mathcal{K})$ to $\ell\left(\mathcal{K}_{\mathcal{S}}\right)$ by Corollary 2.5 , and $\widetilde{H M}\left(W^{\dagger}, \mathfrak{s}\right)(\ell(\mathcal{K}))$ is zero for all $\mathrm{Spin}^{c}$ structures $\mathfrak{s} \neq \mathfrak{s}_{\omega}$. If we restrict $\widetilde{H M}\left(W^{\dagger}\right)$ to the $\operatorname{Spin}^{c}$ structures on $W$ which are extremal with respect to $\bar{R}$ on each component of the boundary, then we have a map

$$
F_{W^{\dagger}}: \operatorname{KHM}(-Y, K) \otimes \mathcal{R} \rightarrow \operatorname{KHM}\left(-Y_{\mathcal{S}}, K_{\mathcal{S}}\right) \otimes \mathcal{R}
$$

such that $F_{W^{\dagger}, \mathfrak{s}}(\ell(\mathcal{K}))$ is $\ell\left(\mathcal{K}_{\mathcal{S}}\right)$ for a unique $\operatorname{Spin}^{c}$ structure (again, $\mathfrak{s}_{\omega}$ ) and zero for all others.

Theorem 5.1 applies to recover an analogue of a theorem of Sahamie [37, Theorem 6.1].

Theorem 5.2 Let $\mathcal{K} \subset(Y, \xi)$ be Legendrian, and let $\left(Y_{ \pm}, \xi_{ \pm}\right)$be the result of a contact $( \pm 1)$-surgery along $\mathcal{K}$. These surgeries induce maps

$$
\begin{aligned}
K H M(-Y, K) \otimes \mathcal{R} & \rightarrow \widetilde{H M}\left(-Y_{+}\right) \otimes \mathcal{R}, \\
\widetilde{H M}\left(-Y_{-}\right) \otimes \mathcal{R} & \rightarrow \operatorname{KHM}(-Y, K) \otimes \mathcal{R}
\end{aligned}
$$

sending $\ell(\mathcal{K}) \mapsto \widetilde{\psi}\left(\xi_{+}\right)$and $\widetilde{\psi}\left(\xi_{-}\right) \mapsto 0$, respectively.

Proof Let $\mathcal{K}^{ \pm}$be Legendrian pushoffs of $\mathcal{K}$ with an extra positive or negative twist around $\mathcal{K}$ as in Figure 5, so that $\mathcal{K}^{+}$(resp. $\mathcal{K}^{-}$) is Legendrian (resp. topologically) isotopic to $\mathcal{K}$. As explained by Ding and Geiges in the proof of [9, Proposition 1], performing a contact $( \pm 1)$-surgery on $\mathcal{K}$ turns $\mathcal{K}^{ \pm}$into a meridian of the surgery torus with $\mathrm{tb}=-1$, so that $\mathcal{K}^{ \pm}$becomes a Legendrian unknot in $\left(Y_{ \pm}, \xi_{ \pm}\right)$. In particular, we have $\operatorname{KHM}\left(-Y_{ \pm}, K^{ \pm}\right) \cong \widetilde{H M}\left(-Y_{ \pm}\right)$and $\ell\left(\mathcal{K}^{ \pm} \subset Y_{ \pm}\right)=\widetilde{\psi}\left(\xi_{ \pm}\right)$. 


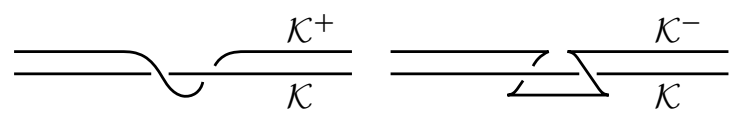

Figure 5. The knots $\mathcal{K}^{+}$and $\mathcal{K}^{-}$are constructed by adding a positive twist and a negative twist, respectively, to a Legendrian pushoff of $\mathcal{K}$.

Writing $\mathcal{S}=\mathcal{K}$ and applying Theorem 5.1 to $(Y, \xi)$, we now have a map

$$
\operatorname{KHM}\left(-Y, K^{+}\right) \otimes \mathcal{R} \rightarrow \operatorname{KHM}\left(-Y_{+}, K^{+}\right) \otimes \mathcal{R}
$$

sending $\ell\left(\mathcal{K}^{+}\right)=\ell(\mathcal{K})$ to $\ell\left(\mathcal{K}^{+} \subset Y_{+}\right)=\widetilde{\psi}\left(\xi_{+}\right)$. Similarly, if we let $\mathcal{S} \subset\left(Y_{-}, \xi_{-}\right)$be the core of the contact $(-1)$-surgery torus, then a contact $(+1)$-surgery on $\mathcal{S}$ cancels the original $(-1)$-surgery, leaving the original contact manifold $(Y, \xi)$. Theorem 5.1 then produces a map

$$
\operatorname{KHM}\left(-Y_{-}, K^{-}\right) \otimes \mathcal{R} \rightarrow \operatorname{KHM}\left(-Y, K^{-}\right) \otimes \mathcal{R}
$$

which sends $\ell\left(\mathcal{K}^{-} \subset Y_{-}\right)=\widetilde{\psi}\left(\xi_{-}\right)$to $\ell\left(\mathcal{K}^{-} \subset Y\right)$. But $\mathcal{K}^{-}$is Legendrian isotopic in $Y$ to the double stabilization $S_{+} S_{-}(\mathcal{K})$, hence $\ell\left(\mathcal{K}^{-} \subset Y\right)=0$ by Proposition 4.2 and we are done.

Corollary 5.3 If the result of contact $(+1)$-surgery on $\mathcal{K} \subset(Y, \xi)$ has nonzero contact invariant $\psi\left(\xi_{+}\right)$, then $\ell(\mathcal{K}) \neq 0$.

Proof Proposition 3.10 provides a map $\widetilde{H M}\left(-Y_{+}\right) \otimes \mathcal{R} \rightarrow \widetilde{H M}\left(-Y_{+}\right) \otimes \mathcal{R}$ sending $\widetilde{\psi}\left(\xi_{+}\right)$to $\psi\left(\xi_{+}\right) \otimes 1$, so if $\psi\left(\xi_{+}\right) \neq 0$ then $\widetilde{\psi}\left(\xi_{+}\right) \neq 0$ and hence $\ell(\mathcal{K}) \neq 0$ as well.

For example, let $K \subset S^{3}$ be a knot with smooth slice genus $g_{s} \geq 1$, and suppose we have a Legendrian representative $\mathcal{K} \subset\left(S^{3}, \xi_{\text {std }}\right)$ of $K$ with $\operatorname{tb}(\mathcal{K})=2 g_{s}-1$. Let $\left(Y_{+}, \xi_{+}\right)$denote the result of contact $(+1)$-surgery on $\mathcal{K}$. The following argument of Lisca and Stipsicz [31], translated directly from Heegaard Floer to monopole Floer homology, shows that $\psi\left(\xi_{+}\right) \neq 0$.

Letting $W$ denote the Weinstein cobordism from $\left(Y_{+}, \xi_{+}\right)$to $\left(S^{3}, \xi_{s t d}\right)$ which reverses the contact $(+1)$-surgery along $\mathcal{K}$, we have a map

$$
\widetilde{H M}\left(-S^{3}\right) \stackrel{F_{W^{\dagger}}}{\longrightarrow} \widetilde{H M}\left(-Y_{+}\right)
$$

sending $\psi\left(\xi_{\text {std }}\right) \neq 0$ to $\psi\left(\xi_{+}\right)$by Corollary 2.5 , so we wish to show that $F_{W^{\dagger}}$ is injective. Now $Y_{+}$is the result of a topological $2 g_{s}$-surgery on $K$, so $-Y_{+}$is the result of a $-2 g_{s}$-surgery on the mirror image $\bar{K}$ and thus $F_{W^{\dagger}}$ fits into a surgery 
exact triangle

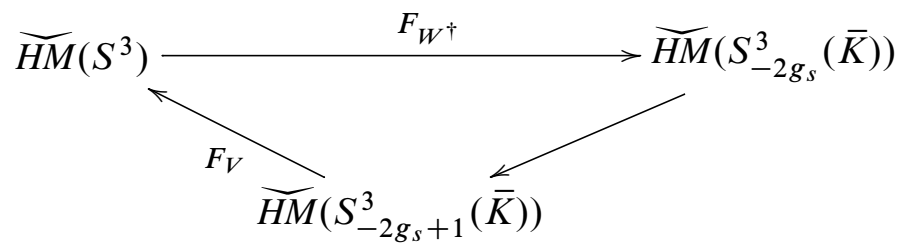

where $V$ is a 2-handle cobordism. Lisca and Stipsicz show that $V$ contains a closed surface $\Sigma$ of genus $g_{s}>0$ and self-intersection $2 g_{s}-1 \geq 0$. If $\mathfrak{s}$ is a $\operatorname{Spin}^{c}$-structure for which $F_{V, \mathfrak{s}} \neq 0$, then the adjunction inequality for cobordisms says that

$$
\left|\left\langle c_{1}(\mathfrak{s}), \Sigma\right\rangle\right|+\Sigma \cdot \Sigma \leq 2 g(\Sigma)-2,
$$

hence $\left|\left\langle c_{1}(\mathfrak{s}), \Sigma\right\rangle\right| \leq-1$, a contradiction. Therefore $F_{V}$ is zero and $F_{W^{\dagger}}$ is injective by exactness.

Corollary 5.4 If $\mathcal{K} \subset\left(S^{3}, \xi_{\text {std }}\right)$ is a Legendrian representative of a knot $K$ with slice genus $g_{s}>0$ and $\operatorname{tb}(\mathcal{K})=2 g_{s}-1$, then $\ell(\mathcal{K}) \neq 0$. Examples include any topologically nontrivial $\mathcal{K}$ for which $\operatorname{tb}(\mathcal{K})=2 g(K)-1$, where $g(K)$ is the Seifert genus of $K$.

For example, in [31] the authors remark that $\overline{\mathrm{tb}}(K)=2 g(K)-1$ for any algebraic knot, where $\overline{\mathrm{tb}}$ denotes maximal Thurston-Bennequin number. More generally, if $\mathcal{K}$ is the Legendrian closure of a positive braid as studied by Kálmán [23] with $n$ strands and $c$ crossings then it is easy to compute that $\operatorname{tb}(\mathcal{K})=c-n=2 g(K)-1$, hence closures of positive braids have the same property.

For any Legendrian knot $\mathcal{K}$, the Legendrian Whitehead double $W(\mathcal{K})$ (due to Eliashberg, and denoted $\Gamma_{\mathrm{dbl}}$ by Fuchs in [17]) is constructed by taking $\mathcal{K}$ and a slight pushoff $\mathcal{K}^{\prime}$ in the $z$-direction, and then replacing a pair of parallel segments with a clasp as in Figure 6; it has genus 1 and tb $=1$.

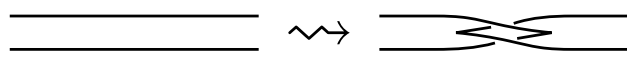

Figure 6. Constructing a Legendrian Whitehead double from $\mathcal{K}$ and its pushoff

Thus $\ell(\mathcal{K}) \neq 0$ if $\mathcal{K}$ is a tb-maximizing representative of the closure of a positive braid or a Legendrian Whitehead double. Similarly, there are many examples of knots with $\operatorname{tb}(\mathcal{K})=2 g_{s}(K)-1$ where $1 \leq g_{s}(K)<g(K)$, and these all have $\ell(\mathcal{K}) \neq 0$; according to KnotInfo [3], the smallest examples have topological types $m\left(8_{21}\right)$ and $m\left(9_{45}\right)$. 


\subsection{Nonloose knots}

By Proposition 4.1, a Legendrian knot $\mathcal{K}$ in an overtwisted manifold is nonloose if $\ell(\mathcal{K}) \neq 0$. Our goal in this section is to apply Theorem 5.1 to construct examples where this is the case. In order to do so, we will first need the following lemma on monopole knot homology and surgery.

Lemma 5.5 Let $K, S \subset Y$ be disjoint knots, and for any integral framing $f$, let $K_{f}$ denote the image of $K$ in the manifold $Y_{f}$ obtained by $f$-surgery along $S$. For each $f$ there is a map $s_{f}: K H M(Y, K) \rightarrow K H M\left(Y_{f}, K_{f}\right)$ corresponding to a 2-handle attachment along $S$ in a closure $\bar{Y}$ of $Y \backslash K$, and these maps satisfy the following:

(1) If $s_{f+1}$ is either injective or surjective, then $s_{f}$ is injective.

(2) If $s_{f}$ is either surjective or zero, then $s_{f+1}$ is zero.

Proof We have a surgery exact triangle

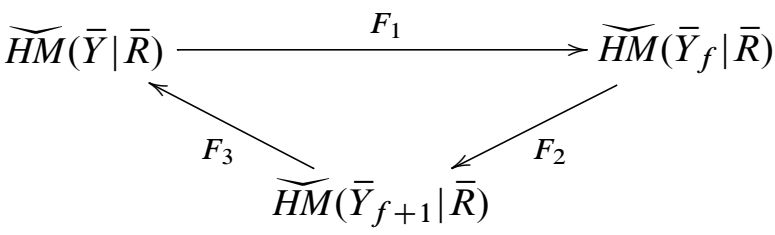

where $\bar{Y}, \bar{Y}_{f}$ and $\bar{Y}_{f+1}$ are closures of the complements of $K$ in $Y, Y_{f}$ and $Y_{f+1}$; note that by definition, $\widetilde{H M}\left(\bar{Y}_{f} \mid \bar{R}\right)=\operatorname{KHM}\left(Y_{f}, K_{f}\right)$. Similarly, we have a second triangle of the form

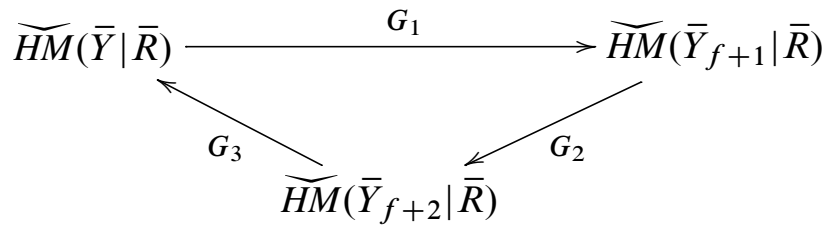

and by [28, Proposition 7.2] we have $G_{1} \circ F_{3}=F_{3} \circ G_{1}=0$, since each composition comes from a cobordism created by a pair of 2-handles which contains a homologically nontrivial sphere of self-intersection zero. This implies that $G_{1}$ (resp. $F_{3}$ ) is zero if $F_{3}$ (resp. $G_{1}$ ) is either injective or surjective.

Suppose $G_{1}$ is either injective or surjective; then $F_{3}=0$, hence by exactness $F_{1}$ is injective. Similarly, if $F_{1}$ is surjective then $F_{2}$ is zero, hence $F_{3}$ is injective, and if $F_{1}$ is zero then $F_{3}$ is surjective; either of these imply $G_{1}=0$. Since $F_{1}=s_{f}$ and $G_{1}=s_{f+1}$, we are done. 
Proposition 5.6 Let $\mathcal{K}, \mathcal{S} \subset(Y, \xi)$ be nullhomologous Legendrian knots such that $S$ is homotopic to a meridian of $K$ in $Y \backslash K$. If $\mathcal{K}_{\mathcal{S}} \subset\left(Y_{\mathcal{S}}, \xi_{\mathcal{S}}\right)$ is the image of $\mathcal{K}$ in the manifold obtained by contact $(+1)$-surgery on $\mathcal{S}$, then $\ell\left(\mathcal{K}_{\mathcal{S}}\right) \neq 0$ if and only if $\ell(\mathcal{K}) \neq 0$ and $\operatorname{tb}(\mathcal{S}) \geq 0$.

Proof Observe that $Y_{\mathcal{S}}$ is obtained from $Y$ by a topological $(\operatorname{tb}(\mathcal{S})+1)$-surgery along $S$, so $-Y_{\mathcal{S}}$ is related to $-Y$ by a $k$-surgery along $S$, where $k=-\operatorname{tb}(\mathcal{S})-1$. In the notation of Lemma 5.5, the map

$$
s_{k}: \operatorname{KHM}(-Y, K) \otimes \mathcal{R} \rightarrow \operatorname{KHM}\left(-Y_{\mathcal{S}}, K_{\mathcal{S}}\right) \otimes \mathcal{R}
$$

carries $\ell(\mathcal{K})$ to $\ell\left(\mathcal{K}_{\mathcal{S}}\right)$ as in Theorem 5.1, so we will show that $s_{k}$ is injective if $k \leq-1$ (ie if $\operatorname{tb}(\mathcal{S}) \geq 0$ ) and zero if $k \geq 0$. By Lemma 5.5 it will be enough to show that $\operatorname{KHM}\left((-Y)_{0}, K_{0}\right)=\widetilde{H M}\left((-\bar{Y})_{0} \mid \bar{R}\right)=0$, where $(\bar{Y}, \bar{R})$ is a closure of $Y \backslash \mathcal{K}$ and $(-\bar{Y})_{0}$ is obtained by 0 -surgery on $S \subset-\bar{Y}$. Indeed, this implies that $s_{k}$ is zero for $k=0$ and hence for all $k \geq 0$, and since $s_{0}$ is also surjective it follows that $s_{k}$ is injective for all $k \leq-1$.

Since $S$ and a meridian of $K$ are homotopic in $Y \backslash K$, they are homotopic in $-\bar{Y}$ as well, and in particular $S$ is homotopic to a nonseparating curve $c \subset \bar{R}$. When we perform 0 -surgery along $S$ to obtain $-\bar{Y}_{0}$, then, the curve $c$ becomes nullhomotopic and so $[\bar{R}] \in H_{2}\left(-\bar{Y}_{0}\right)$ has a representative of genus $g(\bar{R})-1$. Since $g(\bar{R}) \geq 2$, the adjunction inequality tells us that $\widetilde{H M}\left(-\bar{Y}_{0} \mid \bar{R}\right)=0$ as desired.

Corollary 5.7 Let $\mathcal{K} \cup \mathcal{S}$ be a two-component Legendrian link in $\left(S^{3}, \xi_{\text {std }}\right)$ satisfying the following:

(1) $\mathcal{K}$ is a Legendrian unknot with $\operatorname{tb}(\mathcal{K})=-1$.

(2) $\operatorname{tb}(\mathcal{S}) \geq 0$.

(3) The linking number $\operatorname{lk}(K, S)$ is \pm 1 .

Then $\mathcal{K}_{\mathcal{S}}$ is a nonloose Legendrian knot in the contact manifold $\left(S_{\mathcal{S}}^{3}, \xi_{\mathcal{S}}\right)$, where the subscript denotes contact $(+1)$-surgery along $\mathcal{S}$.

Proof We know that $\ell(\mathcal{K})=1 \in \mathcal{R}$ by Proposition 3.8, so Proposition 5.6 tells us that $\ell\left(\mathcal{K}_{\mathcal{S}}\right) \neq 0$.

In particular, given a knot $\mathcal{S}$ with $\operatorname{tb}(\mathcal{S})>0$ which satisfies all the other conditions of Corollary 5.7, we can stabilize $\mathcal{S}$ to get $\mathcal{S}^{\prime}$ with $\operatorname{tb}\left(\mathcal{S}^{\prime}\right) \geq 0$ and then apply the corollary to $\mathcal{K} \cup \mathcal{S}^{\prime}$. Since $\mathcal{S}^{\prime}$ is stabilized, the contact $(+1)$-surgery results in an 
overtwisted contact structure (see for example Ding, Geiges and Stipsicz [10]), and $\mathcal{K}_{\mathcal{S}^{\prime}}$ is nonloose.

For example, the right handed trefoil has a unique Legendrian representative with $(\mathrm{tb}, r)=(1,0)$. Let $\mathcal{S}$ be a stabilization of this Legendrian knot, and let $\mathcal{K}$ be a Legendrian unknot with $\operatorname{lk}(\mathcal{K}, \mathcal{S})= \pm 1$. Then $(+1)$-surgery on $\mathcal{S}$ gives an overtwisted contact structure on the Poincaré homology sphere $-P=-\Sigma(2,3,5)$, which in fact does not admit tight positive contact structures [15], and the image $\mathcal{K}_{\mathcal{S}}$ of $\mathcal{K}$ in $-P$ is a nonloose knot. We exhibit a family $\mathcal{K}_{n}$ of such knots in Figure 7.
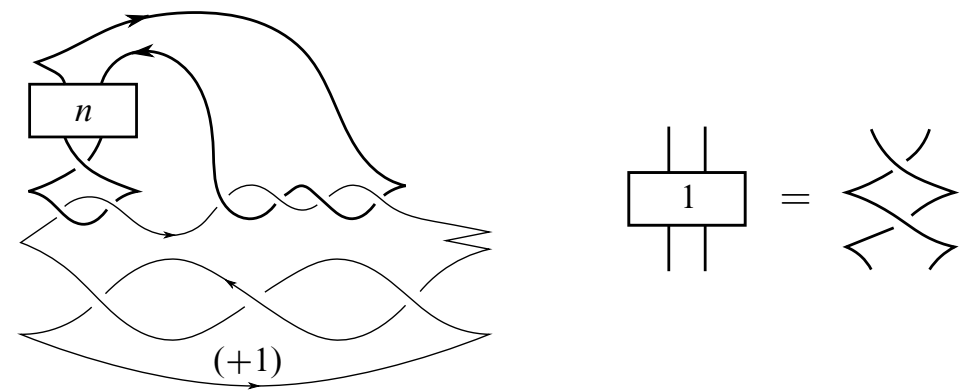

Figure 7. A Legendrian knot $\mathcal{K}_{n}$ in the overtwisted contact structure on $-P$ obtained from $\left(S^{3}, \xi_{\text {std }}\right)$ by contact $(+1)$-surgery on a stabilized righthanded trefoil $\mathcal{T}$

Proposition 5.8 The knots $K_{n}(n \geq 0)$ are all distinct, and none of them are fibered.

Proof Let $L=U_{n} \cup T \subset S^{3}$ and $\hat{L}=K_{n} \cup \widehat{T} \subset-P$, where $\hat{T}$ is the core of the surgery torus glued to $S^{3} \backslash T$ to obtain $-P$. We will compute the Conway polynomial $\nabla_{L}$ and use it to determine $\nabla_{\widehat{L}}$ and $\nabla_{K_{n}}$, and hence the Alexander polynomial $\Delta_{K_{n}}$, referring to the properties stated on page 54 of Boyer and Lines [2]; note that $\nabla$ and $\Delta$ are related by

$$
\nabla_{L}\left(s_{1}, \ldots, s_{m}\right)= \begin{cases}\left(s_{1}-s_{1}^{-1}\right)^{-1} \Delta_{L}\left(s_{1}^{2}\right), & |L|=1, \\ \Delta_{L}\left(s_{1}^{2}, s_{2}^{2}, \ldots, s_{m}^{2}\right), & |L|>1 .\end{cases}
$$

Since $\hat{L}$ is obtained as the cores of surgery tori for (1/0)-surgery on $U_{n}$ and $(1 / 1)-$ surgery on $T$, and $l k\left(U_{n}, T\right)=-1$, the link $\hat{L}$ is determined by $L$ and the framing matrix

$$
B=\left(\begin{array}{cc}
1 & -1 \\
0 & 1
\end{array}\right)
$$

from which we can determine $\mathrm{lk}_{-P}\left(K_{n}, \widehat{T}\right)=-1$ and

$$
\nabla_{\widehat{L}}\left(s_{1}, s_{2}\right)=\nabla_{L}\left(s_{1}, s_{1} s_{2}\right)
$$


by the "variance under surgery" proposition [2, Property (III)]. Then $\nabla_{\hat{L}}(s, 1)=$ $-\left(s-s^{-1}\right) \nabla_{K_{n}}(s)$ by "restriction" [2, Property (IV)], and so

$$
\Delta_{K_{n}}\left(s^{2}\right)=\left(s-s^{-1}\right) \nabla_{K_{n}}(s)=-\nabla_{\widehat{L}}(s, 1)=-\nabla_{L}(s, s) .
$$

Then we have reduced the computation of $\Delta_{K_{n} \subset-P}$ to that of $\nabla_{L \subset S^{3}}(s, s)$. Note that the latter term is determined entirely by a skein relation $\nabla_{L_{+}}-\nabla_{L_{-}}=\left(s-s^{-1}\right) \nabla_{L_{0}}$ :

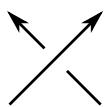

$L_{+}$

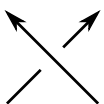

$L_{-}$

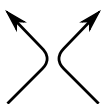

$L_{0}$

and by $\nabla_{U}(s)=\left(s-s^{-1}\right)^{-1}$, where $U$ is the unknot.

Using the skein relation at a crossing in one of the $n$ full twists of $U_{n}$, we see that

$$
\nabla_{U_{n} \cup T}-\nabla_{U_{n-1} \cup T}=\left(s-s^{-1}\right) \nabla_{L_{0}}
$$

and so $\nabla_{L}=\nabla_{U_{0} \cup T}+n\left(s-s^{-1}\right) \nabla_{L_{0}}$. Applying the skein relation to the crossing of $U_{n}$ directly below the $n$ twists, when $n=0$, we get $\nabla_{U_{0} \cup T}-\nabla_{L_{1}}=\left(s-s^{-1}\right) \nabla_{L_{0}}$, hence

$$
\nabla_{L}=\nabla_{L_{1}}+(n+1)\left(s-s^{-1}\right) \nabla_{L_{0}},
$$

where $L=U_{n} \cup T, L_{0}$, and $L_{1}$ are the links in Figure 8.
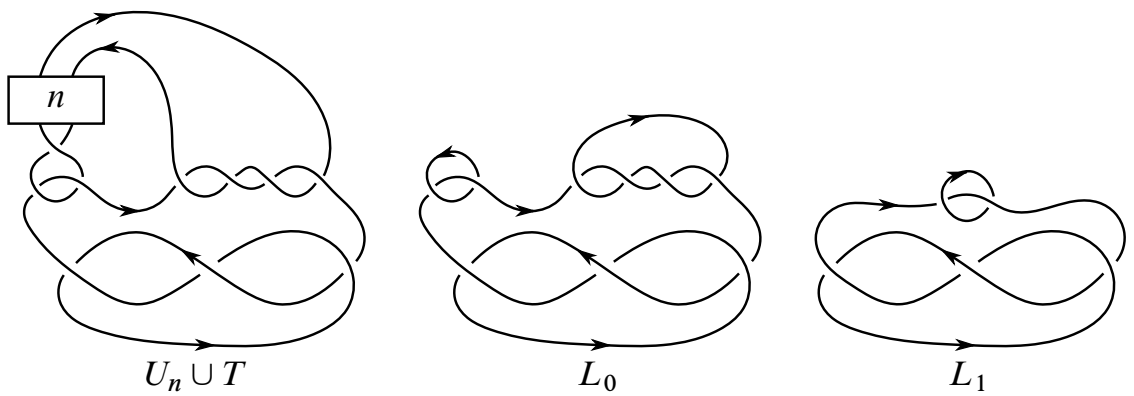

Figure 8. Links appearing in the computation of $L=U_{n} \cup T$ by the skein relation

A straightforward computation now yields

$$
\begin{aligned}
\nabla_{L_{0}}(s, s, s) & =-2\left(s-s^{-1}\right)\left(s^{2}-1+s^{-2}\right), \\
\nabla_{L_{1}}(s, s) & =-\left(s^{2}-1+s^{-2}\right),
\end{aligned}
$$

and since $\Delta_{K_{n}}\left(s^{2}\right)=-\nabla_{L_{1}}-(n+1)\left(s-s^{-1}\right) \nabla_{L_{0}}$ we conclude that

$$
\Delta_{K_{n}}(t)=\left(t-1+t^{-1}\right)\left(1+2(n+1)\left(t-2+t^{-1}\right)\right) .
$$

Since the Alexander polynomials $\Delta_{K_{n}}(t)$ are all distinct, so are the $K_{n}$; and since $\Delta_{K_{n}}$ is never monic, the $K_{n}$ cannot be fibered. 
We remark that in general very few examples of nonloose knots in overtwisted contact manifolds have been studied. Etnyre [13] observed that if the result $\left(S_{\mathcal{K}}^{3}, \xi_{\mathcal{K}}\right)$ of contact (+1)-surgery on $\mathcal{K} \subset\left(S^{3}, \xi_{\text {std }}\right)$ is overtwisted, then the core $\mathcal{K}^{\prime}$ of the surgery torus in $S_{\mathcal{K}}^{3}$ is nonloose. (If $S_{\mathcal{K}}^{3}$ is the Poincaré homology sphere with either orientation, then Ghiggini [18] proved that $K$ must be a trefoil and so one can show that $\Delta_{K^{\prime}}=$ $\pm \Delta_{K} \neq \Delta_{K_{n}}$.) Furthermore, Etnyre and Vela-Vick [16] showed that given an open book decomposition which supports $(Y, \xi)$, any Legendrian approximation of the binding is nonloose. To the best of our knowledge, these are the only known examples in manifolds other than $S^{3}$.

In particular, it seems that the nonloose knots $\mathcal{K}_{n} \subset-P$ were not previously known, and in fact may be the only known nonfibered examples (even in $S^{3}$ ) which are not the cores of surgery tori. The construction of Corollary 5.7 is of course much more general; it would be interesting to give examples of links $\mathcal{K}_{i} \cup \mathcal{S}(i=1,2)$ which are topologically but not Legendrian isotopic and which give distinct nonloose knots $\left(\mathcal{K}_{i}\right)_{\mathcal{S}}$.

\section{Lagrangian concordance}

Chantraine [4] defined an interesting notion of concordance on the set of all Legendrian knots in a contact 3-manifold $Y$.

Definition 6.1 Let $\mathcal{K}_{0}$ and $\mathcal{K}_{1}$ be Legendrian knots parametrized by embeddings $\gamma_{i}: S^{1} \rightarrow Y$, and let $Y \times \mathbb{R}$ be the symplectization of $Y$. We say that $\mathcal{K}_{0}$ is Lagrangian concordant to $\mathcal{K}_{1}$, denoted $\mathcal{K}_{0} \prec \mathcal{K}_{1}$, if there is a Lagrangian embedding $L: S^{1} \times \mathbb{R} \hookrightarrow$ $Y \times \mathbb{R}$ and a $T>0$ such that $L(s, t)=\left(\gamma_{0}(s), t\right)$ for $t<-T$ and $L(s, t)=\left(\gamma_{1}(s), t\right)$ for $t>T$.

Theorem 6.2 [4] The relation $\prec$ descends to a relation on Legendrian isotopy classes of Legendrian knots. If $\mathcal{K}_{0} \prec \mathcal{K}_{1}$ then $\operatorname{tb}\left(\mathcal{K}_{0}\right)=\operatorname{tb}\left(\mathcal{K}_{1}\right)$ and $r\left(\mathcal{K}_{0}\right)=r\left(\mathcal{K}_{1}\right)$.

Our goal in this section is to investigate the behavior of $\ell(\mathcal{K})$ under Lagrangian concordance:

Theorem 6.3 Let $\mathcal{K}_{0}, \mathcal{K}_{1}$ be Legendrian knots in a contact homology 3-sphere $Y$ satisfying $\mathcal{K}_{0} \prec \mathcal{K}_{1}$. Then there is a homomorphism

$$
K H M\left(-Y, K_{1}\right) \otimes \mathcal{R} \rightarrow K H M\left(-Y, K_{0}\right) \otimes \mathcal{R}
$$

sending $\ell\left(\mathcal{K}_{1}\right)$ to $\ell\left(\mathcal{K}_{0}\right)$.

We compare this with the remarks in [4, Section 5.2], where it is observed that Lagrangian concordance induces a map $L C H\left(\mathcal{K}_{1}\right) \rightarrow L C H\left(\mathcal{K}_{0}\right)$ on Legendrian contact homology. 
Proof We fix a particular closure $\left(\bar{Y}_{i}, \bar{R}_{i}\right)$ of the sutured knot complements $Y\left(\mathcal{K}_{i}\right)$ : place the meridional sutures close together so that in $\partial\left(Y \backslash \mathcal{K}_{i}\right)$ they bound an annulus $A$ in which the dividing curves are parallel to a longitude. In the other annulus $A^{\prime}$ bounded by the sutures, the dividing curves twist around the meridional direction a total of $\operatorname{tb}\left(\mathcal{K}_{i}\right)$ times; recall that $\operatorname{tb}\left(\mathcal{K}_{0}\right)=\operatorname{tb}\left(\mathcal{K}_{1}\right)$. We glue a surface $T \times I$ to each complement and round edges, resulting in a manifold with boundary $\bar{R}_{+} \sqcup \bar{R}_{-}$and $\operatorname{int}(A) \subset \bar{R}_{+}$. Finally, we glue $\bar{R}_{+}$to $\bar{R}_{-}$by identifying $(x, 1) \in T \times\{1\}$ to $(x,-1) \in T \times\{-1\}$ for all $x \in \operatorname{int}(T)$, and identifying $A$ with $A^{\prime}$ by a homeomorphism composed of enough Dehn twists around the core of $A$ to make the dividing curves match.

This construction guarantees that $Z_{0}=\bar{Y}_{0} \backslash \operatorname{int}\left(Y \backslash \mathcal{K}_{0}\right)$ and $Z_{1}=\bar{Y}_{1} \backslash \operatorname{int}\left(Y \backslash \mathcal{K}_{1}\right)$ are contactomorphic as 3-manifolds with torus boundary. In the symplectization $Y \times \mathbb{R}$, the cylinder $\mathcal{K}_{0} \times \mathbb{R}$ is Lagrangian, hence it has a standard neighborhood symplectomorphic to a neighborhood $N$ of the 0 -section in $T^{*}\left(S^{1} \times \mathbb{R}\right)$. Then a neighborhood of the boundary $T^{2} \times \mathbb{R}$ of the symplectization $Z_{0} \times \mathbb{R}$, can be identified with the complement of the 0 -section in $N$.

Now consider the Lagrangian cylinder $\mathcal{L} \subset Y \times \mathbb{R}$ defining the concordance from $\mathcal{K}_{0}$ to $\mathcal{K}_{1}$. Once again, $\mathcal{L}$ has a neighborhood symplectomorphic to $N$; if we remove a sufficiently small neighborhood of $\mathcal{L}$, then there is a collar neighborhood of $\partial((Y \times \mathbb{R}) \backslash \mathcal{L})$ which is orientation-reversing symplectomorphic to $N$ with the 0 -section removed. Thus we can glue $(Y \times \mathbb{R}) \backslash \mathcal{L}$ to $Z_{0} \times \mathbb{R}$ to get a symplectic manifold $W$ with two infinite ends. One of these ends is a piece $\bar{Y}_{0} \times(-\infty, T]$ of the symplectization of $\bar{Y}_{0}$, and since $Z_{0}$ is contactomorphic to $Z_{1}$ the other end is $\bar{Y}_{1} \times[T, \infty)$. Thus $W$ is a boundary-exact symplectic cobordism from $\bar{Y}_{0}$ to $\bar{Y}_{1}$.

Finally, we wish to show that the map $i^{*}: H^{1}\left(W, \bar{Y}_{1}\right) \rightarrow H^{1}\left(\bar{Y}_{0}\right)$ is zero. By Poincaré duality it suffices to show that $H_{3}\left(W, \bar{Y}_{0}\right) \rightarrow H_{2}\left(\bar{Y}_{0}\right)$ is zero, or equivalently (by the long exact sequence of the pair $\left.\left(W, \bar{Y}_{0}\right)\right)$ that the map $H_{2}\left(\bar{Y}_{0}\right) \rightarrow H_{2}(W)$ is injective. But there is a natural isomorphism $H_{2}((Y \times \mathbb{R}) \backslash \mathcal{L}) \cong H_{2}\left(Y \backslash \mathcal{K}_{0}\right)$ by Alexander duality, hence by the Mayer-Vietoris sequence and the five lemma it follows that $\mathrm{H}_{2}\left(\bar{Y}_{0}\right) \rightarrow H_{2}(W)$ is an isomorphism as well, and so $i^{*}$ is indeed zero.

Since $W$ is a boundary-exact symplectic cobordism and $H^{1}\left(W, \bar{Y}_{1}\right) \rightarrow H^{1}\left(\bar{Y}_{0}\right)$ is zero, we apply Theorem 2.4 to conclude that

$$
\psi\left(\bar{Y}_{0}, \bar{\xi}_{0}\right)=\widetilde{H M}\left(W^{\dagger}, \mathfrak{s}_{\omega}\right)\left(\psi\left(\bar{Y}_{1}, \bar{\xi}_{1}\right)\right) .
$$

Thus $\widetilde{H M}\left(W^{\dagger}, \mathfrak{s}_{\omega}\right)$ induces a map $f: \operatorname{KHM}\left(-Y, K_{1}\right) \otimes \mathcal{R} \rightarrow \operatorname{KHM}\left(-Y, K_{0}\right) \otimes \mathcal{R}$ satisfying $f\left(\ell\left(\mathcal{K}_{1}\right)\right)=\ell\left(\mathcal{K}_{0}\right)$, as desired. 
Corollary 6.4 If $\mathcal{K}_{0} \prec \mathcal{K}_{1}$ and $\ell\left(\mathcal{K}_{0}\right)$ is nonzero, then so is $\ell\left(\mathcal{K}_{1}\right)$.

Corollary 6.5 If a Legendrian knot $\mathcal{K} \subset\left(S^{3}, \xi_{0}\right)$ bounds a Lagrangian disk in the standard symplectic $4-$ ball $B^{4}$, then $\ell(\mathcal{K})$ is a unit of $\operatorname{KHM}\left(-S^{3}, K\right)$.

Proof The Legendrian unknot $\mathcal{U}$ is Lagrangian concordant to $\mathcal{K}$, and $\ell(\mathcal{U})$ is a generator of $K H M\left(-S^{3}, U\right) \otimes \mathcal{R} \cong \mathcal{R}$ by Proposition 3.8 , so by Theorem 6.3 there is a map $\operatorname{KHM}\left(-S^{3}, K\right) \otimes \mathcal{R} \rightarrow \mathcal{R}$ such that the image of $\ell(\mathcal{K})$ is a unit.

It is observed in the addendum to [4] that the following tangle replacement in the front projection (obtained from a 1-smoothing of a crossing in the Lagrangian projection) can be realized by a Lagrangian saddle cobordism:

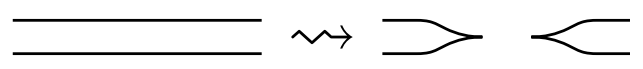

If such a move turns a Legendrian knot $\mathcal{K}$ into a Legendrian unlink whose components are both $\mathcal{U}$, we can cap both components with Lagrangian disks and thus build a Lagrangian slice disk for $\mathcal{K}$, proving that $\ell(\mathcal{K})$ is a primitive element of $K H M\left(-S^{3}, K\right)$. Figure 10 shows grid diagrams for seven such knots, of topological types $m\left(9_{46}\right)$, $m\left(10_{140}\right), m\left(10_{140}\right), 11 n_{139}, m\left(12 n_{582}\right), m\left(12 n_{768}\right)$, and $m\left(12 n_{838}\right)$, which were discovered using a combination of KnotInfo [3], the Legendrian knot atlas [6], and Gridlink [7]. As usual, these may be turned into front projections of Legendrian knots by smoothing out all northeast and southwest corners and then rotating 45 degrees counterclockwise. The dotted line in each diagram indicates where to perform the tangle replacement.

Conjecture 6.6 Given a Lagrangian cobordism $\mathcal{K}_{0} \prec_{\Sigma} \mathcal{K}_{1}$ of arbitrary genus, there is a map $\operatorname{KHM}\left(-Y, K_{1}\right) \otimes \mathcal{R} \rightarrow \operatorname{KHM}\left(-Y, K_{0}\right) \otimes \mathcal{R}$ sending $\ell\left(\mathcal{K}_{1}\right)$ to $\ell\left(\mathcal{K}_{0}\right)$.

\section{References}

[1] J M Bloom, T Mrowka, P Ozsváth, The Künneth principle in Floer homology, in preparation

[2] S Boyer, D Lines, Conway potential functions for links in $\mathbf{Q}$-homology 3-spheres, Proc. Edinburgh Math. Soc. 35 (1992) 53-69 MR1150952

[3] J C Cha, C Livingston, KnotInfo: Table of knot invariants, website (2010) Available at http://www.indiana.edu/ knotinfo

[4] B Chantraine, Lagrangian concordance of Legendrian knots, Algebr. Geom. Topol. 10 (2010) 63-85 MR2580429 

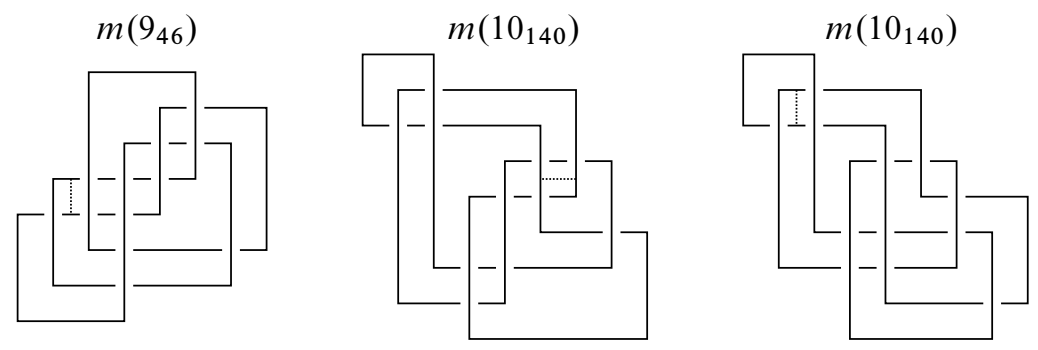

$11 n_{139}$
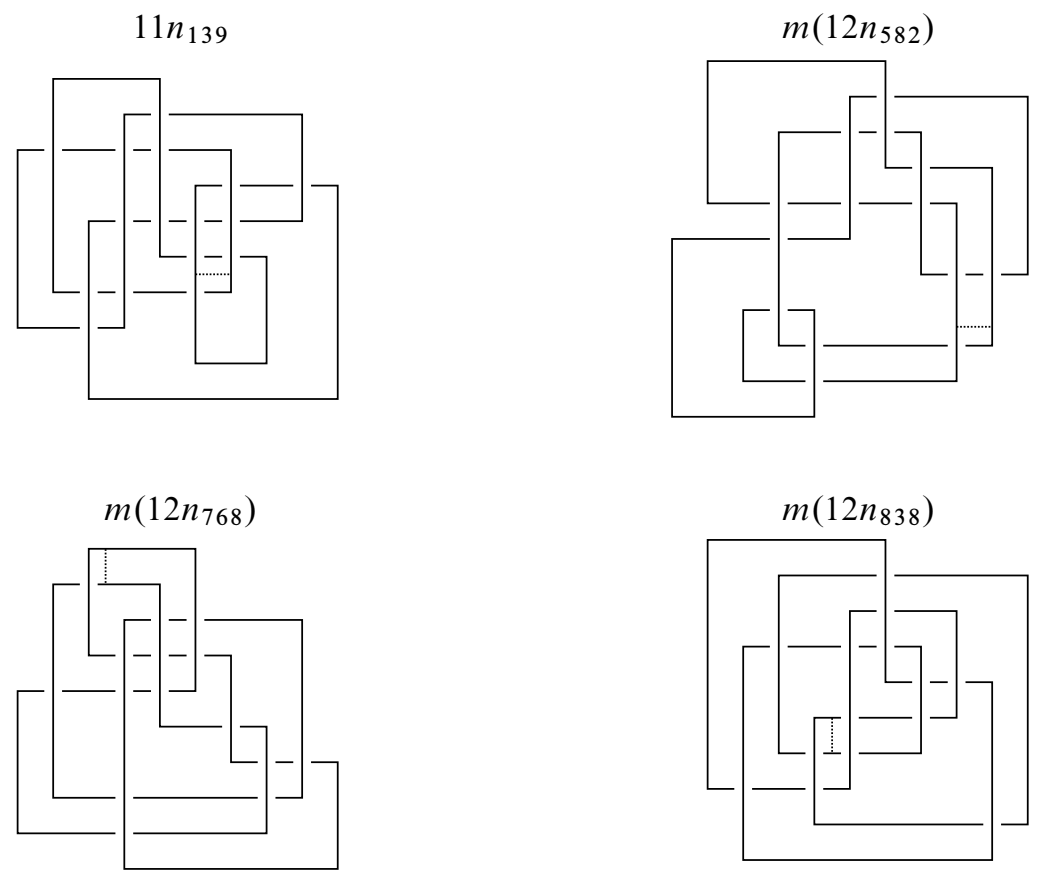

Figure 10. Seven Legendrian knots $\mathcal{K}$ which bound Lagrangian disks in $B^{4}$ and thus satisfy $\ell(\mathcal{K}) \neq 0$

[5] Y Chekanov, Differential algebra of Legendrian links, Invent. Math. 150 (2002) 441483 MR1946550

[6] W Chongchitmate, L Ng, An atlas of Legendrian knots, to appear in Exp. Math. arXiv:1010.3997

[7] M Culler, Gridlink: a tool for knot theorists Available at http:// www.math.uic.edu/ culler/gridlink

[8] F Ding, H Geiges, Symplectic fillability of tight contact structures on torus bundles, Algebr. Geom. Topol. 1 (2001) 153-172 MR1823497 
[9] F Ding, H Geiges, Handle moves in contact surgery diagrams, J. Topol. 2 (2009) 105-122 MR2499439

[10] F Ding, H Geiges, A I Stipsicz, Surgery diagrams for contact 3-manifolds, Turkish J. Math. 28 (2004) 41-74 MR2056760

[11] Y Eliashberg, Invariants in contact topology, from: "Proceedings of the International Congress of Mathematicians, Vol. II (Berlin, 1998)", volume Extra Vol. II (1998) 327-338 MR1648083

[12] Y Eliashberg, M Fraser, Topologically trivial Legendrian knots, J. Symplectic Geom. 7 (2009) 77-127 MR2496415

[13] J B Etnyre, On contact surgery, Proc. Amer. Math. Soc. 136 (2008) 3355-3362 MR2407103

[14] J B Etnyre, K Honda, Knots and contact geometry I: Torus knots and the figure eight knot, J. Symplectic Geom. 1 (2001) 63-120 MR1959579

[15] J B Etnyre, K Honda, On the nonexistence of tight contact structures, Ann. of Math. 153 (2001) 749-766 MR1836287

[16] J B Etnyre, D S Vela-Vick, Torsion and open book decompositions, Int. Math. Res. Not. 2010 (2010) 4385-4398 MR2737776

[17] D Fuchs, Chekanov-Eliashberg invariant of Legendrian knots: existence of augmentations, J. Geom. Phys. 47 (2003) 43-65 MR1985483

[18] P Ghiggini, Knot Floer homology detects genus-one fibred knots, Amer. J. Math. 130 (2008) 1151-1169 MR2450204

[19] E Giroux, Convexité en topologie de contact, Comment. Math. Helv. 66 (1991) 637-677 MR1129802

[20] K Honda, On the classification of tight contact structures I, Geom. Topol. 4 (2000) 309-368 MR1786111

[21] K Honda, W H Kazez, G Matić, Tight contact structures on fibered hyperbolic 3manifolds, J. Differential Geom. 64 (2003) 305-358 MR2029907

[22] A Juhász, Holomorphic discs and sutured manifolds, Algebr. Geom. Topol. 6 (2006) 1429-1457 MR2253454

[23] T Kálmán, Contact homology and one parameter families of Legendrian knots, Geom. Topol. 9 (2005) 2013-2078 MR2209366

[24] Y Kanda, On the Thurston-Bennequin invariant of Legendrian knots and nonexactness of Bennequin's inequality, Invent. Math. 133 (1998) 227-242 MR1632790

[25] P B Kronheimer, T S Mrowka, Monopoles and contact structures, Invent. Math. 130 (1997) 209-255 MR1474156

[26] P Kronheimer, T Mrowka, Monopoles and three-manifolds, New Math. Monogr. 10, Cambridge Univ. Press (2007) MR2388043 
[27] P Kronheimer, T Mrowka, Knots, sutures, and excision, J. Differential Geom. 84 (2010) 301-364 MR2652464

[28] P Kronheimer, T Mrowka, P Ozsváth, Z Szabó, Monopoles and lens space surgeries, Ann. of Math. 165 (2007) 457-546 MR2299739

[29] Y Lekili, Heegaard Floer homology of broken fibrations over the circle arXiv: 0903.1773

[30] P Lisca, P Ozsváth, A I Stipsicz, Z Szabó, Heegaard Floer invariants of Legendrian knots in contact three-manifolds, J. Eur. Math. Soc. 11 (2009) 1307-1363 MR2557137

[31] P Lisca, A I Stipsicz, Ozsváth-Szabó invariants and tight contact three-manifolds I, Geom. Topol. 8 (2004) 925-945 MR2087073

[32] T Mrowka, Y Rollin, Legendrian knots and monopoles, Algebr. Geom. Topol. 6 (2006) 1-69 MR2199446

[33] T Mrowka, Y Rollin, Contact invariants and monopole Floer homology, in preparation

[34] K Niederkrüger, C Wendl, Weak symplectic fillings and holomorphic curves, Ann. Sci. Éc. Norm. Supér. (4) 44 (2011) 801-853

[35] P Ozsváth, A I Stipsicz, Contact surgeries and the transverse invariant in knot Floer homology, J. Inst. Math. Jussieu 9 (2010) 601-632 MR2650809

[36] P Ozsváth, Z Szabó, D Thurston, Legendrian knots, transverse knots and combinatorial Floer homology, Geom. Topol. 12 (2008) 941-980 MR2403802

[37] B Sahamie, Dehn twists in Heegaard Floer homology, Algebr. Geom. Topol. 10 (2010) 465-524 MR2602843

[38] A I Stipsicz, V Vértesi, On invariants for Legendrian knots, Pacific J. Math. 239 (2009) 157-177 MR2449016

[39] C H Taubes, Embedded contact homology and Seiberg-Witten Floer cohomology V, Geom. Topol. 14 (2010) 2961-3000 MR2746727

[40] C Wendl, A hierarchy of local symplectic filling obstructions for contact 3-manifolds arXiv: 1009.2746

Department of Mathematics, Harvard University

Cambridge, MA 02138, USA

ssivek@math.harvard.edu

http://math.harvard.edu/ ssivek

Proposed: Peter S. Ozsváth

Seconded: Yasha Eliashberg, Ronald J. Stern 